\title{
Diabatic and Adiabatic Representations for Atomic Collision Processes
}

John B. Delos

William \& Mary, jbdelos@wm.edu

W. R. Thorson

Follow this and additional works at: https://scholarworks.wm.edu/aspubs

Part of the Physics Commons

\section{Recommended Citation}

Delos, John B. and Thorson, W. R., Diabatic and Adiabatic Representations for Atomic Collision Processes (1979). Journal of Chemical Physics, 70(4).

https://doi.org/10.1063/1.437650

This Article is brought to you for free and open access by the Arts and Sciences at W\&M ScholarWorks. It has been accepted for inclusion in Arts \& Sciences Articles by an authorized administrator of W\&M ScholarWorks. For more information, please contact scholarworks@wm.edu. 


\title{
Diabatic and adiabatic representations for atomic collision processes $^{\text {a) }}$
}

\author{
J. B. Delos \\ Physics Department, College of William and Mary, Williamsburg, Virginia 23185
}

W. R. Thorson

Chemistry Department, University of Alberta, Edmonton, Alberta, Canada T6G 262

(Received 15 September 1978)

\begin{abstract}
A consistent general definition of diabatic representations has not previously been given, even though many practical examples of such representations have been constructed for specific problems. Such a definition is provided in this paper. Beginning with a classical trajectory formulation, we describe the form and behavior of velocity-dependent couplings in slow collisions, including the effects of electron-translation factors (ETF's). We compare the couplings arising from atomic representations and atomic ETF's with those arising from molecular representations and "switching function" ETF's. We show that a unique set of switching functions makes the two descriptions identical in their effects. We then show that an acceptable general definition of a diabatic representation is provided by the condition $P+A=0$, where $P$ is the usual nonadiabatic coupling matrix and $A$ represents corrections to it arising from electron translation factors (ETF's). Two distinct types of diabatic representation result, depending on the definition taken for $A$. States that undergo no deformation are called F-diabatic; those that have no velocity-dependent couplings are called M-diabatic. Finally, we discuss the properties of representations that are partially diabatic and partially adiabatic, and we give some rules for the construction of representations that should be nearly optimal for describing many types of collision processes.
\end{abstract}

\section{INTRODUCTION}

The intuitive idea of a diabatic representation of atomic collision processes is a very old one, dating back at least to Zener's classic paper on the curve crossing problem ${ }^{1}$; in that paper, he assumed that the basis functions being used were approximate eigenfunctions of the molecular electronic Hamiltonian, but that they did not have the sudden rapid "change of character" that is typical of exact molecular eigenstates near a crossing or avoided crossing. Thirty years later, such representations were given the name "diabatic" by Lichten, ${ }^{2}$ the word being chosen to suggest that the states do not adiabatically adjust to the instantaneous position of the nuclei. Such states have been defined and used to study a great variety of processes; in any given situation, intuitive physical reasoning has always produced a useful basis set with the desired properties. ${ }^{3-9}$

On the other hand, a general formal definition of diabatic states has been lacking. An attempt at such a definition was made earlier by F. T. Smith ${ }^{10}$ : For radial problems, defining

$$
P_{i j}^{R}(R)=\int \phi_{i}^{*}(\mathbf{r} ; R)\left\{-i \hbar d / d R\left[\phi_{j}(\mathbf{r} ; R)\right]\right\} d \mathbf{r}
$$

[where $\mathbf{r}$ collectively denotes the set of electronic coordinates, $R$ is the internuclear distance, and $\left\{\phi_{j}(\mathbf{r} ; R)\right\}$ is a set of electronic basis functions that may or may not depend parametrically on $R$ ], he proposed that a diabatic basis is one in which $P^{R}$ vanishes. This defini-

\footnotetext{
a) Preliminary reports of this work were presented at the Annual Meeting of the Division of Electron and Atomic Physics, American Physical Society, Lincoln, Nebraska (1976), and at the International Conference on the Physics of Electronic and Atomic Collisions, Paris (1977).
}

tion is very close to the correct one given in this paper, but it has an apparently serious formal defect ${ }^{5 \mathrm{c}, 11}$ : If, for a given $j$, the scalar product of $d \phi_{j}(r ; R) / d R$ with $\phi_{i}$ vanishes for all $i$ in a complete set, then $\left(d \phi_{j} / d R\right)$ must itself be zero. If every matrix element $P_{i j}^{R}(R)$ vanishes for a complete set, then every state in that set must be completely independent of $R$. Not only do such basis states have none of the distortion, polarization, and change of character with changing $R$ that is typical of molecular electronic states, but also they do not even translate along with the nuclei.

A rationale for Smith's proposal might be found in the following physical argument: Adiabatic molecular electronic states make the electronic Hamiltonian diagonal at each nuclear configuration $R$; transitions between such states are produced, in a quantum description, by the nuclear kinetic energy operator, or, in a classical trajectory description, by the time-derivative operator

$$
i \hbar \partial / \partial t=i \hbar \mathrm{v} \cdot \nabla_{R} \text {. }
$$

Under appropriate conditions, ${ }^{12}$ transition probabilities induced by the time dependence of adiabatic states must tend to zero at low velocities, and the system then actually follows the adiabatic behavior described by the adiabatic states. At higher velocities, however, the electrons do not have time to adjust to the changing molecular potential, and, in a sudden collision, the actual electronic wave function of the system may remain nearly fixed in character. Described in terms of adiabatic basis states, this "nonchange" of the system wave function appears as a transition. Diabatic basis states have been sought because they can more nearly represent actual system behavior at finite collision velocities. Since it is the change in character of adiabatic states with $R$ which is responsible for the "transitions," then- 
insofar as such change of character is represented by the $d / d R$ operator - we can construct a suitable diabatic representation by making the matrix elements of $d / d R$ zero or negligibly small.

There is only one flaw in this argument, and we have already alluded to it: $P^{R}$ represents not only the effects of any change of character of basis functions, but also the effects of their simple translation with the moving nuclear centers. Since we want our diabatic states to move along with the nuclei, we need a representation in which at most that portion of $P$ representing polarization, distortion, and change of character of basis functions is made to vanish.

The most general means for doing this became clear to us through the quantum mechanical formulation of the theory of electron translation factors (ETF's). ${ }^{13}$ We showed that the usual coupled equations of molecular collision theory lead to some incorrect results, and that the problems arise because the basis set (or, in an alternative formulation, the coordinate system) does not properly describe the translation of the electron along with the nuclei. When ETF's are included (or the coordinate system is modified), the most important result is that the matrix $P$ appearing in the coupled equations is replaced by a corrected matrix $(P+A)$, where $A$ is defined below [cf. Eqs. (III. 8d)]. $A$ is a matrix which identifies and cancels that portion of $P$ that represents merely the translation of basis functions along with moving nuclei. Accordingly, $(P+A)$ is the part of $P$ representing actual change of character, distortion, or polarization of the basis - the part that really is responsible for nonadiabatic transitions. Therefore, an appropriate formal definition of diabatic states can be given by the general relation

$$
P+A=0 \quad \text { (or negligibly small). }
$$

The purpose of this paper is to develop this idea and its consequences. We will show that there are several types of states which are diabatic in this sense; the nature of these states depends upon the scheme used to specify the correction matrix $A$, and upon whether all vector components of $\mathbf{P}+\mathbf{A}$ are made to vanish or only the radial part [Eq. (I.2) is deliberately ambiguous on these points]. Moreover, since Eq. (I. 2) might be made to hold only within a truncated manifold of basis states, and then perhaps only approximately, it is clear that the description of a state as diabatic usually has a relative, rather than an absolute, meaning: A state might be diabatic with respect to some particular physical interaction or set of interactions, but be adiabatic with respect to others. In practice, this last point is well understood, and an important conclusion of this paper is that the intuitive methods used to construct diabatic representations in the past can be justified quite rigorously.

On the other hand, we can also show that the prescription (I. 2) meets the formal requirements of any desired level of rigor or completeness, and that one set of basis states which satisfies Eq. (I.2) exactly is just a set of atomic states which move along with their atomic nuclei but undergo no real physical change. As a further con- sequence, we can show that-given a definite connection between adiabatic molecular electronic states and the atomic orbitals used to construct them, such as is normally obtained in a quantum chemical calculation $-a$ unique prescription of the corrected nonadiabatic coupling matrix $(\mathbf{P}+\mathbf{A})$ for an adiabatic representation is then obtained. Studies of this formula and of its relevance for the problem of selecting optimum switching functions for molecular states ${ }^{13,14}$ are topics outside the scope of this paper and are pursued in other forthcoming work. ${ }^{15}$

In this paper, we develop the theory starting from the classical trajectory formulation of atomic collision theory. While this involves some approximations, these are well understood, ${ }^{16,17}$ and in this form the theory is so transparent as to make the main results of the paper seem almost trivial. Similar results can be developed within a fully quantum mechanical formulation.

The plan of this paper is as follows: We begin by treating in detail only the one-electron case, since this contains all the essential features of the formal problem. In Sec. II, we define three types of electronic basis functions commonly used in collision calculations, and we define their associated electron-translation factors. In Sec. III, the classical trajectory equations are put into a simplified form which is suitable for slow collisions; we pay particular attention to the effects of the ETF's on the couplings. We then consider the properties of the coupled equations under basis set transformations in the limit of low collision velocity. In particular, we show that if we start from a description based on a set of atomic orbitals (effectively, diabatic states), carry out a transformation to a set of adiabatic states by making the electronic Hamiltonian diagonal at each $R$, and impose the requirement of physical invariance upon the resulting description in adiabatic representation, then we obtain an unique definition of the coupling matrix $(P+A)$ in terms of the transformation matrix linking the two descriptions. Section IV then proceeds to the major formal results of this paper: the definitions of diabatic representations and the consequences of these definitions. Having established these definitions clearly for the one-electron case, we then discuss in a qualitative way the additional concepts or generalizations needed to apply the same ideas to multielectron systems. Finally, we turn our attention to a separate but closely related question. Given a formal definition of diabatic representations, is there some procedure or prescription that may help us to find an optimal representation for any given collision process? A cautiously affirmative answer to this question is given in Sec. $\mathrm{V}$.

\section{ELECTRONIC BASIS SETS FOR ATOMIC COLLISION THEORY}

\section{A. Definitions of basis types}

We will consider three classes of electronic basis states, but we require all of them to have certain general properties:

(1) The basis states are assumed to depend on time $t$ only through their dependence on the internuclear vector $\mathbf{R}(t)$. 
(2) We assume that the basis states are square integrable, representing bound electronic states, and for simplicity we take them to be real.

(3) We assume that, as $R \rightarrow \infty$, at least some of the basis states correspond to atomic eigenstates representing the open channels of interest.

(4) For the moment, we assume that basis states are defined in the rotating molecular frame of reference (later, we briefly consider some nonrotating basis states).

For simplicity, we consider first only the one-electron case.

We can take the geometric center (midpoint between the two nuclei) as the origin for electron coordinates. ${ }^{18}$ Let $\rho=\left(\rho_{x}, \rho_{y}, \rho_{z}\right)$ be the electron coordinate expressed in a space-fixed (i.e., nonrotating) frame, and $\mathbf{r}=(x$, $y, z)$ the same vector expressed in the rotating molecular frame, whose polar axis is $R(R, \theta, \Phi)$. We write the basis functions as $\phi(\mathrm{r} ; R)$ when expressed in molecular coordinates; $\hat{\phi}(\rho ; R)$ denotes the same rotating basis function expressed in the space fixed coordinate system

$$
\hat{\phi}(\rho ; \mathbf{R})=\phi(\mathbf{r} ; R) \text {. }
$$

It can be shown that ${ }^{13}$

$$
\begin{aligned}
& -i \hbar \partial / \partial R[\hat{\phi}(\rho ; \mathbf{R})]=-i \hbar \partial / \partial R[\phi(\mathbf{r} ; R)], \\
& -i \hbar \partial / \partial \Theta[\hat{\phi}(\rho ; \mathbf{R})]=-L_{y} \phi(\mathbf{r} ; R), \\
& -i \hbar \partial / \partial \Phi[\hat{\phi}(\rho ; \mathbf{R})]=\left(\sin \Theta L_{x}-\cos \Theta L_{\mathbf{z}}\right) \phi(\mathbf{r} ; R) .
\end{aligned}
$$

Now let us define the three classes of basis states.

\section{Class F states ("fixed one-center orbitals")}

A basis function $\phi_{n}(\mathrm{r} ; R)$ is said to be in class $F$ if there exists a constant $\kappa_{n}$ such that $\phi_{n}(\mathbf{r} ; R)$ depends upon $R$ only as

$$
\phi_{n}(r ; R)=\phi_{n}\left(x, y, z-\kappa_{n} R\right) .
$$

Class $F$ includes all basis functions that rotate with the molecular frame and are carried along with some center (a nucleus, or the geometric center, or center of mass) but otherwise have no change whatever. We may say that they have no "intrinsic" $R$ dependence, but only the "extrinsic" $R$ dependence implied by Eq. (II. 3).

With a class $F$ state can be associated a single center ETF: Defining the velocity $\mathbf{v}=(d \mathbf{R} / d t)$, we write

$$
\hat{F}_{n}=\exp \left[(i m / \hbar)\left\{\mathrm{v} \cdot \kappa_{n} \rho-\frac{1}{2} \int^{t} \kappa_{n}^{2} \mathrm{v}^{2} d t^{\prime}\right\}\right] .
$$

This factor describes the momentum and kinetic energy of an electron as it is carried along with a center moving at velocity $\kappa_{n} v$ (with respect to the geometric center). Normally, we consider only class $F$ states that are centered on the nuclei, i. e., $\kappa_{n}= \pm \frac{1}{2}$.

\section{Class V states ("variable one-center orbitals")}

States in class $V$ may have additional parameters, such as orbital exponents, that are allowed to vary smoothly with $R$, but like the class $F$ states, these states can be associated with a specific center with which they propagate. Class $V$ states can be written in the form

$$
\phi_{n}\left[\mathbf{r}-\kappa_{n} R ; \zeta_{1}(R), \ldots, \zeta_{J}(R)\right],
$$

where $\zeta_{1}, \ldots, \zeta_{J}$ are parameters (e.g., orbital exponents) which may vary slowly with $R$. These states are also moving with the definite velocity $\kappa_{n} v$, and so the appropriate ETF is again of the single-center form (II. 4). It is convenient to regard class $F$ states as a special case of class $V$ states; in class $F$, the parameters $\zeta_{1}, \ldots, \zeta_{J}$ are fixed and independent of $R$.

\section{Class $M$ states ("molecular orbitals")}

Class $M$ states are distinguished from the previous two classes in that they are essentially molecular in character; the electron is shared by the two nuclei, and there is no single center with which the electron can be said to be propagating. Examples of such states are the $g$ and $u$ molecular orbitals for the Born-Oppenheimer states of a homonuclear diatomic system, and the valence electron orbitals for such heteronuclear systems as $(\mathrm{Li}-\mathrm{Na})^{+}$, which are intrinsically molecular for $R \approx 10$ a. u. Class $M$ basis states also include molecular orbitals which need not be eigenstates of any particular Hamiltonian; the only essential property is their twocenter character. ${ }^{19}$ For such states, single-center ETF' $s$ of the form (II. 4) are not appropriate because a class $M$ orbital as a whole does not have any single velocity of propagation.

Instead, the ETF for class $M$ states is constructed using a local propagation velocity for an electron in such an orbital. ${ }^{13,14,20}$ This is done by defining a switching function $f_{n}(r ; R)$, which varies smoothly as a function of electron position $r$; typically, it may approach -1 near nucleus $A$, and +1 near nucleus $B$, and these limiting values must hold as $R \rightarrow \infty$. Like the basis functions, the switching function is defined in the rotating molecular frame but can be re-expressed in space-fixed variables as in Eq. (II. 1):

$$
\hat{f}_{n}(\rho ; \mathbf{R})=f_{n}(\mathbf{r} ; R) \text {. }
$$

Using the switching function, we define a local propagation velocity for an electron in orbital $n$ :

$$
\mathbf{w}_{n}(\rho ; \mathbf{R})=\frac{1}{2} \hat{f}_{n}(\rho ; \mathbf{R}) \mathbf{v} \text {; }
$$

then we take the ETF to be $\mathrm{e}^{21}$

$$
\hat{F}_{n}=\exp \left[(i m / \hbar)\left\{\mathbf{w}_{n}(\rho ; \mathbf{R}) \cdot \rho-\frac{1}{8} \int^{t} \mathbf{v}^{2} d t^{\prime}\right\}\right] .
$$

In the kinetic energy term, we have replaced $\mathrm{w}_{n}^{2}$ by its limiting asymptotic value $\frac{1}{4} \mathrm{v}^{2}$; it turns out that this makes certain of the velocity dependent couplings smaller than when the local choice is used.

An ETF for class $V$ (or $F$ ) states can be regarded as a special case of this more general ETF; we just take $f_{n}(r ; R)$ to be a constant, with $\kappa_{n}=\frac{1}{2} f_{n}$, and Eq. (ПI. 8) reduces to Eq. (II. 4).

In some cases, it may be possible to use the same switching function for all of the basis states $\left\{\phi_{n}\right\}$ in a class $M$ basis. When this is possible, it is very convenient, because then $F_{k}^{*} F_{n}=1$ for all $k$ and $n$ in the set. 
However, in this paper, we do not assume that all switching functions are necessarily the same.

\section{B. Completeness vs sufficiency}

We would like to be able to assume that the basis functions form a complete set, but there are two problems. First, although a set of molecular eigenfunctions may be formally complete (provided the continuum is included), no real calculation ever makes use of a complete set. Second, the set of all discrete and continuous atomic orbitals based on two different centers is in principle overcomplete and nonorthogonal, but in practice incomplete, nonorthogonal, and sometimes nearly linearly dependent (in a large two-center atomic basis set, the overlap matrix $S$ may become nearly singular). To deal with these problems, formal completeness is abandoned in favor of convenience and accuracy; this is especially evident in cases where "pseudostates" are used. Such problems are very familiar to valence theorists and they appear in the same way in collision theory.

So, although we cannot assume that the basis sets are really complete, we will assume that they are sufficient to obtain a description of the required accuracy. In addition, there are certain points in our development at which we assume that the sets used are large enough and near enough to complete sets that the closure relation

$$
1=\sum_{n}\left|\phi_{n}\right\rangle\left\langle\phi_{n}\right|
$$

for orthogonal basis states, or more generally

$$
\begin{aligned}
& 1=\sum_{k, n}\left|\phi_{k}\right\rangle S_{k n}^{-1}\left\langle\phi_{n}\right|, \\
& S_{k n}=\left\langle\phi_{k} \mid \phi_{n}\right\rangle,
\end{aligned}
$$

holds to sufficient accuracy. General use of a closure condition must be viewed with caution in any real calculation, but here we will only use it in such a way that the result will be accurate if the basis states are reasonably accurate eigenfunctions of the Hamiltonian, no matter how sharply the adiabatic set is then truncated.

\section{COUPLED EQUATIONS FOR SLOW COLLISIONS}

A. Classical trajectory equations in the low velocity limit

Assuming that the nuclei move along a classical path, the Schrödinger equation for the electrons is

$$
h \Upsilon=i \hbar(\partial \Upsilon / \partial t) \text {, }
$$

where as usual the potential energy term in the electronic Hamiltonian $h$ is time dependent because of the nuclear motion. We choose a basis set of one of the above types, denoting the corresponding electronic ket $|n\rangle$ $-\phi_{n}(r ; R)$. The appropriate type of ETF is appended and the state vector is expanded in the ETF-modified set

$$
\Upsilon=\sum_{n} d_{n}(t) F_{n}|n\rangle .
$$

To obtain coupled equations, we put Eq. (III. 2) into (III. 1), multiply on the left by $\langle k| F_{k}^{*}$, and rearrange terms to obtain

$$
\begin{gathered}
\sum_{n}\left\langle k\left|F_{k}^{*} F_{n}\right| n\right\rangle\left\{i \hbar d / d t\left[d_{n}(t)\right]\right\}=\sum_{n}\left(\left\langle k\left|F_{k}^{*} F_{n}\left(h-i \hbar \mathrm{v} \cdot \nabla_{R}\right)\right| n\right\rangle\right. \\
\left.+\left\langle k\left|F_{k}^{*}\left\{\left[h, F_{n}\right]-i \hbar \partial F_{n} / \partial t\right\}\right| n\right\rangle\right) d_{n}(t) .
\end{gathered}
$$

Within the limits of the basis set, these equations are equivalent to the Schrödinger equation (III. 1), and within the approximations inherent in the classical trajectory theory, we may say they are "exact."

For some simple problems, ${ }^{22-26}$ it is possible to evaluate the matrix elements required and obtain direct numerical solutions to Eq. (III. 3). However, the matrix elements are explicitly velocity dependent, and exact evaluation of terms in Eq. (III. 3) leads to some complications that are not relevant to slow collisions. We will simplify the equations using two approximations:

(1) In evaluating the term in curly brackets in $\mathrm{Eq}$. (III. 3), we will ignore the acceleration $d v / d t$ and also those residual terms of order $v^{2}$ which arise from $d \hat{f}_{n} / d t=\mathrm{v} \cdot \nabla_{R} \hat{f}_{n}$ and from the difference between local and asymptotic transport kinetic energies $(m / 8)\left(\hat{f}_{n}^{2}-1\right) \mathrm{v}^{2}$. (These approximations are exact if only "single-center" ETF's and a rectilinear trajectory are used.) The coupled equations then take the form

$S(\mathrm{v})[i \hbar d / d t d(t)]=\{h(\mathrm{v})+\mathrm{v} \cdot[\mathbf{P}(\mathrm{v})+\mathbf{A}(\mathrm{v})]\} d(t)$,

where

$$
\begin{aligned}
& S_{k n}(\mathrm{v})=\left\langle k\left|F_{k}^{*} F_{n}\right| n\right\rangle, \\
& h_{k n}(\mathbf{v})=\left\langle k\left|F_{k}^{*} F_{n} h\right| n\right\rangle, \\
& \mathbf{P}_{k n}(\mathbf{v})=\left\langle k\left|F_{k}^{*} F_{n}\left(-i \hbar \nabla_{R}\right)\right| n\right\rangle, \\
& \mathbf{A}_{k n}(\mathbf{v})=(i m / \hbar)\left\langle k\left|F_{k}^{*} F_{n}\left[h, \mathbf{s}_{n}\right]\right| n\right\rangle, \\
& \mathbf{s}_{n}=\frac{1}{2} f_{n}(\mathbf{r} ; R) \mathbf{r} .
\end{aligned}
$$

Because of the neglected terms in $(d v / d t)$ and $\mathbf{v}^{2}$, Eqs. (III. 4) may not exactly conserve probability in general, but this failure of unitarity is small, of the order of the neglected terms. If desired, exactly unitary equations can be obtained by reinstating the neglected terms or by other more artificial devices. Actually, our main reason for making this approximation is so that the final coupled equations will be manifestly analogous to those obtained from our quantum formulations. ${ }^{13}$

(2) The second approximation entails a more substantial simplification, but it is appropriate only for slow collisions. Expanding the ETF's in a power series, we neglect all terms of order $v^{2}$ and higher:

$$
\begin{aligned}
& S(\mathbf{v})=s+\mathbf{v} \cdot \boldsymbol{\sigma}+\cdots, \\
& h(\mathbf{v})=h+\mathbf{v} \cdot \eta+\cdots, \\
& \mathbf{P}(\mathbf{v})=\mathbf{P}+\cdots, \\
& \mathbf{A}(\mathbf{v})=\mathbf{A}+\cdots,
\end{aligned}
$$

where

$$
\begin{aligned}
& S_{k n}=\langle k \mid n\rangle, \\
& h_{k n}=\langle k|h| n\rangle, \\
& \mathbf{P}_{k n}=\left\langle k\left|-i \hbar \nabla_{R}\right| n\right\rangle, \\
& \mathbf{A}_{k n}=(i m / \hbar)\left\langle k\left|\left[h, \mathbf{s}_{n}\right]\right| n\right\rangle
\end{aligned}
$$


are the zero-velocity limits, and

$$
\begin{aligned}
& \sigma_{k n}=(i m / \hbar)\left\langle k\left|\left(\mathbf{s}_{n}-\mathbf{s}_{k}\right)\right| n\right\rangle, \\
& \eta_{k n}=(i m / \hbar)\left\langle k\left|\left(\mathbf{s}_{n}-\mathbf{s}_{k}\right) h\right| n\right\rangle .
\end{aligned}
$$

[Note that since $P(v)$ and $A(v)$ are multiplied by $v$ in $E q$. (III.4), we retain only the zero-velocity limit of these matrices in Eqs. (III. 7c) and (III. 7d).] Applying these approximations to Eqs. (III. 4), we obtain

$$
(S+\mathrm{v} \cdot \sigma) i \hbar d / d t d=[h+\mathrm{v} \cdot \eta+\mathrm{v} \cdot(\mathbf{P}+\mathbf{A})] d .
$$

A second form has comparable accuracy and may be more convenient; expanding

$$
(S+\mathbf{v} \cdot \sigma)^{-1}=S^{-1}-S^{-1} \mathbf{v} \cdot \sigma S^{-1}+\cdots,
$$

then

$$
i \hbar d / d t d=S^{-1}[h+\mathbf{v} \cdot(\mathbf{P}+\mathbf{A})+\mathbf{v} \cdot \gamma] d,
$$

where

$$
\gamma=\eta-\sigma S^{-1} h .
$$

Though Eqs. (III. 10) and (III. 12) are not identical, both are accurate to order $\mathrm{v}$, and either can be used. We work mostly with Eq. (III. 12) in the rest of this paper.

\section{B. Properties of the coupled equations}

\section{Magnitude and interpretation of various terms}

Let us now examine the terms obtained in Eqs. (III. 12) by this low-velocity approximation. The two zero-order terms $S i \hbar d$ and $h$ and the first-order term $\mathbf{v} \cdot \mathbf{P}$ are the standard terms which arise in the simplest form of the "perturbed stationary states" theory, in which ETF's are neglected. The $\mathrm{v} \cdot \mathrm{A}$ term arises from the action of the operator $h$ on the ETF's; as was mentioned in the introduction, $\mathbf{A}$ identifies and cancels that part of $\mathbf{P}$ that merely represents displacement of basis functions. Although matrix elements of $\mathbf{P}$ can become locally very large in special situations (such as curve crossings described in an adiabatic representation), in general $\mathbf{P}$ and $\mathrm{A}$ are comparable in magritude, and in a very fundamental sense they "belong together."

The remaining first-order terms, which we have collectively denoted by $\gamma$, arise from the expansion of the "momentum transfer factor" $F_{k}^{*} F_{n}$ in powers of $\mathrm{v}$; from the definitions (III.9), it can be seen that the individual terms $\eta$ and $\sigma S^{-1} h$ can be comparable in magnitude to $\mathrm{A}$. However, we can show that the total result $\gamma$ either vanishes or is small in certain cases:

(i) $\gamma$ always goes to zero as $R-\infty$, because $S^{-1}$ and $h$ then connect only states $(k, n)$ associated with a common center and $\left(\boldsymbol{s}_{n}-\boldsymbol{B}_{k}\right)$ then vanishes.

(ii) $\eta$ and $\sigma S^{-1} h$ each vanish separately if the same ETF is used to describe all states. This is not appropriate for class $V$ states arising from two centers, but in a class $M$ description it is sometimes acceptable to use a common switching function.

(iii) If the basis states are eigenfunctions of $h, \gamma$ vanishes at all values of $R$. Further, if the basis states are such that all but a small part of $h$ is diagonal, then it may be possible to neglect $v \cdot \gamma$ since it is proportional both to $\mathrm{v}$ and to the (assumed small) off-diagonal portion of $h$. However, for a class $V$ (or $F$ ) basis, where $h$ typically has substantial off-diagonal elements, $\gamma$ cannot be shown to be negligible, and it should be included in a proper calculation.

It might be questioned whether the expansion in powers of $v$ was carried out in a consistent manner: In particular, since

$$
i \hbar d / d t d=i \hbar \mathrm{v} \cdot \nabla_{R} d,
$$

it might appear that this term is first order in $v$, and that quantities like $(\mathrm{v} \cdot \sigma)\left(\mathrm{v} \cdot \nabla_{R} d\right)$ are terms of second order in $v$ that should be neglected in a consistent development. This appearance is deceiving: $v \cdot \nabla_{R} d$ does not go to zero as $v$ goes to zero; instead $\left|\nabla_{R} d\right|$ goes to infinity in such a way that $\mathrm{v} \cdot \nabla_{R} d$ remains finite. Hence, while terms like $\left[\boldsymbol{v} \cdot \nabla_{R}(\mathbf{v} \cdot \sigma)\right]$ which arise below are of order $v^{2},(\mathrm{v} \cdot \sigma)\left(\mathbf{v} \cdot \nabla_{R} d\right)$ is only of order $v$.

Finally, let us note here a relation that will be useful later. If we are using a class $F$ or class $V$ basis, so that the ETF $F_{n}$ has the single-center form (II. 4), then it follows directly from Eq. (III. 8d) that

$$
\left(\mathrm{A}_{F}\right)_{k n}=\mathrm{p}_{k n} \kappa_{n}
$$

this can also be written formally as

$$
\mathrm{A}_{F}=\mathbf{p} S^{-1} \kappa,
$$

where $\mathrm{p}$ is the matrix representing the electronic momentum

$$
\mathrm{p}_{k n}=\int \phi_{k}^{*}(\mathbf{r} ; R)\left(-i \hbar \nabla_{r}\right) \phi_{n}(\mathrm{r} ; R) d^{3} \mathbf{r}
$$

and $\kappa$ is the matrix whose elements are defined

$$
\kappa_{k n}=\left\langle\phi_{k}\left|\kappa_{n}\right| \phi_{n}\right\rangle=S_{k n} \kappa_{n} .
$$

In Eq. (III. 14), we have denoted such an $\mathrm{A}$ matrix by the subscript $F$ (or $V$ ), to sharply distinguish it from A's arising from class $M$ ETF's $\left(\mathbf{A}_{M}\right)$, which do not possess the property (III. 14b) (except for certain cases such as $R-\infty)$.

\section{Probability conservation}

The coupled equations given above do not exactly conserve probability, but the unitarity errors are of order $v^{2}$, like the intrinsic error in the equations themselves. This is most easily seen from the form (III. 10), using an analysis first given by $\mathrm{Green}^{27}$ :

$$
\begin{aligned}
& i \hbar d / d t\langle\Upsilon \mid \Upsilon\rangle=i \hbar d / d t\left[d^{\dagger} S(v) d\right] \\
& \simeq i \hbar d / d t\left[d^{\dagger}(S+\mathbf{v} \cdot \sigma) d\right] \\
&=d^{\dagger}\left\{[h+\mathbf{v} \cdot(\eta+\mathbf{P}+\mathbf{A})]-\left[h^{+}+\mathbf{v} \cdot\left(\eta^{\dagger}+P^{\dagger}+A^{\dagger}\right)\right]\right. \\
&+i \hbar d / d t(S+\mathbf{v} \cdot \sigma)\} d .
\end{aligned}
$$

Now, $h$ is a Hermitian matrix, as is the combination $\eta+\mathrm{A}$; also, it is easily shown that

$$
\mathbf{P}-\mathbf{P}^{\dagger}=-i \hbar \nabla_{R} S,
$$

so all but one of the terms in Eq. (III. 16) cancel, leaving

$$
i \hbar d / d t\langle\Upsilon \mid \Upsilon\rangle=d^{\dagger}\left[\mathbf{v} \cdot \nabla_{R}(\mathrm{v} \cdot \sigma)\right] d=\mathcal{O}\left(v^{2}\right) .
$$




\section{Alternative form}

There is an alternative form of the coupled equations (III. 12) which might be especially useful in computations. If we define

$$
\rho_{k n}=(i m / \hbar)\left\langle k\left|s_{n}\right| n\right\rangle
$$

and

$$
\left(\rho^{\dagger}\right)_{k n}=-(i m / \hbar)\left\langle k\left|\mathbf{s}_{k}\right| n\right\rangle
$$

so that

$$
\sigma=\rho+\rho^{\dagger},
$$

and we invoke the closure relation (II. 10) to simplify $A$ and $\eta$, then we obtain

$$
i \hbar d / d t d=S^{-1}[h+\mathrm{v} \cdot(\mathrm{P}+\mathrm{C})] d,
$$

where

$$
\mathrm{C}_{k n}=\left(h S^{-1} \rho-\rho S^{-1} h\right)_{k n} .
$$

Equations (III. 21) have the computational advantage that all low-velocity effects of ETF's are contained in the single matrix $\rho$. However, our main concerns in this paper are formal and for this purpose we continue to focus attention upon Eqs. (III. 12).

\section{Separation of radial from angular couplings}

If the nuclear momentum operator in the $\mathbf{P}$ matrix (III. 8c) is expressed in spherical coordinates, then the angular parts of the gradient can be evaluated using Eq. (II. 2); this gives the standard separation of radial from angular couplings ${ }^{10,13}$

$$
\mathbf{P}_{k n}=\hat{e}_{R} P_{k n}^{R}+\hat{e}_{\Theta} P_{k n}^{\Theta}+\hat{e}_{\Phi} P_{k n}^{\Phi},
$$

where $\hat{e}_{R}, \hat{e}_{\Theta}$, and $\hat{e}_{\Phi}$ are the unit vectors for spherical coordinates, and

$$
\begin{aligned}
& P_{k n}^{R}=\langle k|-i \hbar \partial / \partial R| n\rangle, \\
& P_{k n}^{\ominus}=-R^{-1}\left\langle k\left|L_{y}\right| n\right\rangle, \\
& P_{k n}^{\Phi}=R^{-1}\left\langle k\left|L_{x}-\cot \theta L_{z}\right| n\right\rangle .
\end{aligned}
$$

The corresponding components of $A$ are obtained by expressing $\mathbf{s}_{n}$ in the rotating molecular frame

$$
\begin{aligned}
& \mathbf{A}_{k n}=\hat{e}_{R} A_{k n}^{R}+\hat{e}_{\Theta} A_{k n}^{\ominus}+\hat{e}_{\varphi} A_{k n}^{\Phi}, \\
& A_{k n}^{R}=(i m / \hbar)\left\langle k\left|\left[h, s_{n}^{z}\right]\right| n\right\rangle, \\
& A_{k n}^{\ominus}=(i m / \hbar)\left\langle k\left|\left[h, s_{n}^{x}\right]\right| n\right\rangle, \\
& A_{k n}^{\ominus}=(i m / \hbar)\left\langle k\left|\left[h, s_{n}^{y}\right]\right| n\right\rangle,
\end{aligned}
$$

and a similar decomposition holds for $\sigma$ and $\eta$. Now, in most applications, the nuclear trajectory is assumed to be in a plane $\Phi=$ constant, so $v_{\Phi}=0$, and the $\Phi$ components of these matrices can be ignored. The coupled equations, therefore, take the form

$$
\begin{aligned}
i \hbar d / d t d= & {\left[S^{-1} h+S^{-1} v^{R}\left(P^{R}+A^{R}\right)+S^{-1} v^{\Theta}\left(-L_{y} / R+A^{\Theta}\right)\right.} \\
& \left.+S^{-1} \mathrm{v} \cdot\left(\eta-\sigma S^{-1} h\right)\right] d .
\end{aligned}
$$

\section{Change of representation}

We shall define a general change of representation in the following way: Suppose Eqs. (III.12) have been derived using a set of basis states $\left\{\phi_{n}^{1}(\mathrm{r} ; R)\right\}$ to which ap- propriate ETF's have been appended in the usual way; then further suppose that a second set of basis states spanning the same space $\left\{\phi_{\mu}^{2}(\mathbf{r} ; R)\right\}$ is related to the first set by a transformation $U$ :

$$
\left|\phi_{\mu}^{2}\right\rangle=\sum_{n} U_{n \mu}\left|\phi_{n}^{1}\right\rangle
$$

( $U$ is not necessarily unitary, but is certainly invertible, and may be a function of $R$ ). Then, it follows that the matrices $S, h, \mathbf{P}$, and $\mathbf{p}$, which consist of matrix elements of operators that are defined "a priori," i.e., independent of representation, transform as

$$
M^{2}=U^{\dagger} M^{1} U
$$

except for $P$, which obeys the rule

$$
\mathbf{P}^{2}=U^{\dagger} \mathbf{P}^{1} U-i \hbar U^{\dagger} S^{1} \nabla_{R} U \text {. }
$$

The other matrices which appear in the theory, i.e., $\mathrm{A}, \eta$, and $\sigma$ (or $\rho, \mathrm{C}$, and $\kappa$ ), are not composed of matrix elements of operators definable in a representationindependent way, since ETF's are always defined in connection with some particular representation [in this case, the basis $\left.\left\{\phi_{n}^{1}(r ; R)\right\}\right]$. These matrices may nevertheless be transformed according to Eq. (III. 29) as well. Finally, if we also define transformations of the coefficients $d$ according to

$$
d^{2}=U^{-1} d^{1}
$$

then under such invertible transformations the form of Eqs. (III. 12) remains invariant.

Such a change of representation does not alter the physical content of the original system of equations, but it may change the way we describe that content. If the coupled equations are to be solved numerically, the effort required for a given number of states is not very sensitive to the choice of representation. However, in some cases, a change of representation can lead to further simplifying approximations or physical insight. For instance, the number of coupled states needed to describe a slow collision might be dramatically reduced using an adiabatic representation; on the other hand, collisional autoionization and electron detachment are more easily described in some sort of diabatic representation. ${ }^{8,28}$

\section{Connection between atomic and molecular descriptions}

We have formulated coupled equations for slow collisions using class $V$ or class $F$ states, with single-center ETF's, and using class $M$ states, with molecular ETF's based on switching functions. Although we have done this in a way which preserves the formal resemblance of the two types of description, and results in coupled equations of the same general form (III.12), we have not established any necessary a priori connection between the two types of description, and in general no such connection need exist. This situation arises because the switching functions used to construct a class $M$ basis state description may be chosen in a quite arbitrary way, even when the class $M$ basis states $\left\{\left|\phi_{\mu}^{M}\right\rangle\right\}$ themselves are fully specified. For class $V$ or class $F$ descriptions, no corresponding ambiguity exists, be- 
cause for a single-center function there is no doubt at all about the choice of ETF required to correct exactly for the simple translation of the basis state with the moving center.

This arbitrariness in a class $M$ description is not a source of formal difficulty; if the basis set used really were complete, then calculated cross sections would be independent of the choice of switching functions. However, for a finite basis, the results may well depend upon the chosen $f_{\mu}$ 's. Therefore, an important problem for class $M$ descriptions is to find a set of switching functions that provides the most accurate possible cross sections for a given (finite) basis set $\left\{\left|\phi_{\mu}^{M}\right\rangle\right\}$.

In certain cases, experience has shown ${ }^{14}$ that it is possible to choose some simple $f_{\mu}$ 's which are "optimal" in the sense that they produce a systematic cancellation of a large portion of the uncorrected matrix $\mathbf{P}$. In many cases, the residual couplings are reduced by several orders of magnitude. Since the effects observed are systematic and lead to quite definite choices for $f_{\mu}$, such studies could provide one way of selecting ETF's for class $M$ states. However, such an approach is computationally very cumbersome and so far has been applied only to the separable one-electron two-center problem.

The fact that no ambiguity exists in a class $V$ or class $F$ description suggests that we might establish a definite choice for switching functions in a class $M$ description by requiring actual as well as formal invariance of the description to basis transformations of the sort described in the preceding subsection. Such a connection can in fact be made, and is accurate to first order in $v$, which is the stated accuracy of Eqs. (III. 12) themselves.

Suppose we have an arbitrary set of class $M$ electronic basis states $\left\{\left|\phi_{\mu}^{N}\right\rangle\right\}$, and this is related to a set of class $V$ (or class $F$ ) basis states $\left\{\left|\phi_{n}^{V}\right\rangle\right\}$ by an invertible transformation $U$ :

$$
\left|\phi_{\mu}^{M}\right\rangle=\sum_{n} U_{n \mu}\left|\phi_{n}^{V}\right\rangle
$$

Now suppose further that we associate with the basis state $\left|\phi_{\mu}^{M}\right\rangle$ a "special" switching function $f_{\mu}$ such that

$$
\mathbf{s}_{\mu}\left|\phi_{\mu}^{M}\right\rangle=\sum_{n} U_{n \mu} \mathbf{s}_{n}\left|\phi_{n}^{V}\right\rangle ;
$$

since $\boldsymbol{s}_{n}$ is unambiguously defined, it follows that $\boldsymbol{s}_{\mu}$ is also defined, given the matrix $U$.

The state vector $\Upsilon$ may be written [cf. Eq. (III.2)]

$$
\Upsilon^{V}=\sum_{n} d_{n}^{V} F_{n}^{V}\left|\phi_{n}^{V}\right\rangle
$$

in the class $V$ description; in the class $M$ description, it is given by

$$
\Upsilon^{M}=\sum_{\mu} d_{\mu}^{M} F_{\mu}^{M}\left|\phi_{\mu}^{M}\right\rangle
$$

If we choose $\boldsymbol{s}_{\mu}\left|\phi_{\mu}^{M}\right\rangle$ according to the special prescription (III. 33), then it is easy to show, by expanding the ETF's in powers of $v$, that $\Upsilon^{v}=\Upsilon^{M}$ to first order in $v$, i.e., $\Upsilon$ is invariant to the transformation (III. 32) within errors $\mathcal{O}\left(v^{2}\right)$. Moreover, since Eq. (III. 32) implies the matrix transformations described in Sec. III. B. 5, we also have that

$$
\rho_{M}=U^{\dagger} \rho_{V} U,
$$

where

$$
\left(\rho_{M}\right)_{\nu \mu}=(i m / \hbar)\left\langle\phi_{\nu}^{M}\left|\mathbf{s}_{\mu}\right| \phi_{\mu}^{M}\right\rangle
$$

and

$$
\left(\rho_{V}\right)_{k n}=(i m / \hbar)\left\langle\phi_{k}^{V}\left|\boldsymbol{s}_{n}\right| \phi_{n}^{V}\right\rangle .
$$

The same result holds also for $\sigma$ and $C$ (and trivially for $S, h$, etc. ). We can also write

$$
\frac{1}{2}\left\langle\phi_{\nu}\left|f_{\mu}\right| \phi_{\mu}\right\rangle=\left(U^{\dagger} \kappa U\right)_{\nu \mu} \text {. }
$$

This invariance of identity under transformation does not hold, however, for A, nor for $\eta$; only their sum has this property

$$
\left(\mathbf{A}_{M}+\eta_{M}\right)=U^{\dagger}\left(\mathbf{A}_{V}+\eta_{V}\right) U
$$

where the matrices $A_{M}$ and $\eta_{M}$ are defined using Eqs. (III. 8d) and (III. 9b), respectively, with $\mathbf{s}_{\mu}$ given by Eq. (III. 33). The mixing implied in Eq. (III. 38) again emphasizes that we must always distinguish sharply between matrices $A_{M}$ and matrices $A_{V}$ (or $A_{F}$ ) and never assume that one goes into the other under any transformation.

A further important result applies if the transformation $U$ is such that the states $\left\{\left|\phi_{\mu}^{M}\right\rangle\right\}$ are eigenfunctions of $h$ (adiabatic representation). In that case, we proved that

$$
\gamma_{M}=\eta_{M}-\sigma_{M} S^{-1} h=0 ;
$$

hence, if we use the connection (III. 33), we obtain

$$
\mathbf{A}_{\mu}=U^{\dagger}\left(\mathbf{A}_{V}+\gamma_{V}\right) U=U^{\dagger}\left(A_{V}+\eta_{V}-\sigma_{V} S^{-1} h\right) U
$$

if the $M$ basis is adiabatic. Since we know that $\gamma_{V}$ does not vanish (except as $R \rightarrow \infty$ ), this special case shows explicitly that $A_{M}$ contains more than just $A_{V}$, a significant point for the problem of diabatic states.

These "special" switching functions defined by Eq. (III. 33) provide a very specific identification of the "displacement part" of the $R$ dependence in molecular states. Preliminary evidence suggests that the switching functions calculated in this way agree well with those calculated by the much more difficult "optimization" studies cited earlier. An explicit study and applications of this definition of the ETF corrections will be presented in a forthcoming paper. ${ }^{15}$ In our present work, we do not necessarily wish to assume that such a specific choice for class $M$ state descriptions has been made, and most of our results have a general validity independent of the form chosen for $\mathbf{s}_{\mu}$.

\section{FORMAL DEFINITION OF DIABATIC STATES}

\section{A. Overview}

We proposed Eq. (I. 2) as a general definition of a diabatic representation, but noted that its meaning is ambiguous. In this section, we examine different specific interpretations which can be given to this definition. First, we distinguish between applications of Eq. (I. 2) to the radial component $\left(P^{R}+A^{R}\right)$ only, and those which 
include all vector components. Second, in Sec. III, we showed that a sharp distinction must be made between matrices of type $A_{V}$ or $A_{F}$ (based on single-center ETF's), and those of type $A_{M}$ (constructed explicitly using class $M$ states and ETF's based on switching functions); a corresponding distinction must be made between diabatic representations for which $\left(P^{R}+A_{F}^{R}\right)=0$ (type " $F$ ") and those for which $\left(P^{R}+A_{\mu}^{R}\right)=0$ (type “ $M^{\prime \prime}$ ). Third, Eq. (I. 2) is also ambiguous in that it might hold only within a sharply truncated set of states, or only hold approximately.

Even though many important applications of the theory of diabatic representations occur for many-electron systems, the essential formal characteristics of the problem appear for the one-electron case, which is treated in detail. In Sec, IV. B. 4, we give a brief indication how the theory can be extended to the many-electron case. Given an appropriate extended definition of ETF's and their associated matrices $A$ and $\gamma$, all definitions and conclusions of this section remain valid.

\section{B. Systems with electrostatic couplings only}

The main interest in the problem of diabatic states, and also the case where their meaning and definition has been most elusive, is the case where only the radial components $\left(P^{R}+A^{R}\right)$ are relevant, and we devote most of our discussion to this case; a brief discussion of angular couplings and the generalization of Eq. (I. 2) to all vector components $(P+A)$ is given in Sec. IV. D. We may further restrict the detailed discussion to those cases where only the electrostatic part of $h$ (the part that neglects spin-orbit coupling and smaller magnetic effects) plays any role in the problem (the case of spinorbit coupling is treated separately in Sec. IV.C). Couplings $\left(P^{R}+A^{R}\right)$ and/or any off-diagonal parts of $h$ then connect states of the same diatomic molecular symmetry. The expression "radial coupling" has commonly been applied to such cases, but "electrostatic coupling" is more precise and descriptive.

The coupled equations for such a problem take the form

$$
i \hbar d / d t d=S^{-1}\left[h+v^{R}\left(P^{R}+A^{R}+\gamma^{R}\right)\right] d,
$$

where

$$
\gamma^{R}=\eta^{R}-\sigma^{R} S^{-1} h
$$

and we can der ive such equations in different representations and from different starting points.

\section{Adiabatic (Born-Oppenheimer) representation}

For these systems, the adiabatic representation is always defined by the orthogonal set of eigenstates of $h$. The eigenstates, eigenvalues, and sometimes the important elements of $P^{R}$ are routinely calculated by quantum chemists. As suggested earlier, the re are then two ways to obtain coupled equations of the form (IV.1): (a) Since the quantum chemical calculation typically begins with a class $V$ (or possibly a class $F$ ) basis, and the ETF's proper to such a basis are given in Eq. (II. 4), the matrices $A_{V}^{R}$ and $\gamma_{V}^{R}$ can be computed in the original representation of $V$ or $F$ states and then transformed into the adiabatic representation according to Eqs.
(III. 29) and (III. 30), respectively, by the same matrix $U(R)$ which diagonalizes $h$; then we have

$$
i \hbar d / d t d^{\prime}=\left[h^{\prime}+v^{R}\left(P^{R^{\prime}}+A_{v}^{R^{\prime}}+\gamma_{v}^{R^{\prime}}\right)\right] d^{\prime},
$$

where the prime denotes the matrices after transformation to adiabatic representation. (b) Alternatively, once given the adiabatic eigenvectors of $h$ and the matrix $P^{R}$, switching functions may be chosen for each adiabatic state and the matrix $A_{M}^{R}$ is then computed directly in adiabatic representation, using Eq. (III. 8d); then we have

$$
i \hbar d / d t d=\left[h+v^{R}\left(P^{R}+A_{M}^{R}\right)\right] d
$$

(recall that, in a basis where $h$ is diagonal, $\gamma_{M}^{R}$ vanishes).

We repeat that Eqs. (IV. 3a) and (IV. 3b) are not necessarily identical within a finite basis, because no constraint has been placed on the choice of switching functions used to define $A_{M}^{R}$. Only if the special switching functions of Eq. (III. 33) are used is $A_{\mu}^{R}$ equal to $A_{V}^{R^{\prime}}+\gamma_{V}^{R^{\prime}}$. Moreover, even in that case, $A_{V}^{R^{\prime}}$ is not to be identified with $A_{M}^{R}$ because the invariance condition (III. 38) only holds for $A^{R}+\eta^{R}$. We denote by $A_{\mu}^{R}$ a matrix $A$ computed originally in a (usually adiabatic) molecular representation using switching functions, and any transform of such a matrix according to Eq. (III. 29). $A_{F}^{R}$ or $A_{V}^{R}$ means any transform of an $A$ matrix that was computed originally in a class $F$ or $V$ basis with singlecenter ETF's.

\section{Diabatic representations}

We admit several different types of representations to the class of diabatic representations. We will say that a representation is diabatic if all, or a part, of the matrix $\left(P^{R}+A^{R}\right)$ either vanishes or is negligible: The representation is fully diabatic if $P^{R}+A^{R}$ vanishes exactly in a complete set; it is partially diabatic if a selected subset $\{(m, n)\}$ of elements of $\left(P^{R}+A^{R}\right)$ vanish; and it is approximately diabatic if the relevant portion of $\left(P^{R}+A^{R}\right)$ can be considered "negligibly small." This terminology deliberately does not specify whether $A^{R}$ is of type $A_{F}^{R}$ or type $A_{H}^{R}$.

$F$-diabatic representations. An $F$-diabatic representation is defined by the condition $\left(P^{R}+A_{F}^{R}\right)=0$. The properties of such representations follow from the simple and obvious theorem:

$$
\left[(\partial / \partial R)_{r}+\kappa_{n}(\partial / \partial z)_{R}\right] \phi_{n}(r ; R)=0
$$

if and only if

$$
\phi_{n}(\mathrm{r} ; R)=\phi_{n}\left(x, y, z-\kappa_{n} R\right),
$$

i. e., if and only if $\phi_{n}$ is in class $F$. Since $P^{R}$ is just the matrix of $-i \hbar \partial / \partial R$, and $A_{F}^{R}$ is either the matrix of $\left(-i \hbar \kappa_{n} \partial / \partial z\right)$, or a transform of it, we have the following consequences:

(1) If every state in a representation is in class $F$, then $P^{R}+A_{F}^{R} \equiv 0$, i. e., a class $F$ representation is fully $F$ diabatic.

(2) If any particular state $\phi_{k}(r ; R)$ in a representation is in class $F$, then the $k$ th column of $\left(P^{R}+A_{F}^{R}\right)$ vanishes.

(3) Any representation obtained by an $R$-independent 
invertible transformation from a representation based on class $F$ states is also fully diabatic. (Hence, not every fully $F$-diabatic state is necessarily a class $F$ state.)

(4) Conversely to (1), if in some complete set we find that $P^{R}+A_{F}^{R}=0$ and that $A_{F}^{R}$ satisfies Eqs. (III. 14), i.e.,

$$
\left(A_{F}^{R}\right)_{k n}=-i \hbar\left\langle\phi_{k}\left|\kappa_{n} \partial / \partial z\right| \phi_{n}\right\rangle \quad(\text { all } k, n),
$$

then every state in that representation is in class $F$.

(5) Conversely to (2), if in some complete set we find that the entire $k$ th column of $P^{R}+A_{F}^{R}$ vanishes, and that

$$
\left(A_{F}^{R}\right)_{j k}=\kappa_{k}\left(p_{k}\right)_{j k},
$$

then $\phi_{k}(r ; R)$ is in class $F$.

Fully $F$-diabatic states meet one of the intuitive criteria that would be expected for "diabatic states": These states move along with the nuclei to which they are attached, but do not change character in any way. But on the other hand, we have shown that these states have nonvanishing velocity-dependent radial couplings $v^{R} \gamma^{R}$ and also they are not orthogonal.

$M$-diabatic representations. An $M$-diabatic representation is defined by the condition $\left(P^{R}+A_{M}^{R}\right)=0$. Basis states for this type of representation have detailed properties that depend on the switching function used to calculate $A_{M}^{R}$; and in any case they will not generally be the same as $F$-diabatic states.

As $R \rightarrow \infty, \frac{1}{2} f_{n}(\mathbf{r} ; R)$ assumes the proper value for $\kappa_{n}$ near each center, and we know that $\gamma_{F}^{R}$ goes to zero. Therefore, $A_{\mu}^{R}$ and $A_{F}^{R^{\prime}}$ become identical and the conditions $\left(P^{R}+A_{F}^{R}\right)=0$ and $\left(P^{R}+A_{m}^{R}\right)=0$ become equivalent. $M$-diabatic states must therefore either become class $F$ states, or fixed linear combinations of such states, in the limit $R \rightarrow \infty$. (Of course, this result is rather trivial, since such an asymptotic correspondence to class $F$ states holds for the basis states of any reasonable representation.)

For symmetric systems, switching functions must have ungerade symmetry, and Eqs. (III. 12) can have no couplings between $g$ and $u$ states. For such systems, them, $M$-diabatic states retain the $g$ or $u$ symmetry and the two-center molecular character of the adiabatic states.

The really significant property of $M$-diabatic states is that all velocity-dependent couplings in the transformed equations are made to be zero (or negligibly small). Hence, the coupled equations in an $M$-diabatic representation have the form intuitively desired of a diabatic description. Although $M$-diabatic states are not class $F$, and must undergo some (presumably slow) change with $R$, and although their detailed nature depends upon the choice of switching functions used to define $A_{M}^{R}$, this detailed behavior is of no concern to us if we are only interested in the actual solution of a collision problem: To solve the coupled equations, one only needs to know the matrix elements of $h$ and $S$ in the diabatic representation.

$f_{n}(\mathbf{r} ; R)$ independent of $n$. One especially simple and convenient $M$-diabatic representation is obtained if we take the same switching function for all states, because then $A_{N}^{R}$ is Hermitian. To find the diabatic representation, we then require $U(R)$ such that

$$
U^{\dagger}\left(P^{R}+A_{M}^{R}\right) U-i \hbar U^{\dagger} d U / d R=0 .
$$

Since $\left(P^{R}+A_{M}^{R}\right)$ is Hermitian, it easily follows that $d / d R\left(U^{\dagger} U\right)=0$, i.e., $U^{\dagger} U$ is a constant. However, since we know that $\left(P^{R}+A_{R}^{R}\right)-0$ as $R-\infty$, we can choose $U(R-\infty)=1$ as our initial condition; then $U^{\dagger}(R) U(R)=1$, $U(R)$ is unitary, and the diabatic basis states are orthogonal. The coupled equations then take the especially simple form

$$
i \hbar d / d t d=h d \text {. }
$$

\section{Construction of diabatic representations}

The definitions and properties given above suggest several methods for constructing diabatic representations; which method is appropriate depends upon the objectives of the problem, the required accuracy, and the amount of information available from earlier stages in the calculation.

Partial or total decomposition of a known adiabatic construction. As noted earlier, a quantum chemist typically begins the construction of an adiabatic state description with a set of basis functions based on atomic states or atomic orbitals; these may be class $F$ states, but more commonly they are class $V$ states. This basis is usually quite large compared to the number of adiabatic states calculated. Then the Born-Oppenheimer Hamiltonian is made diagonal by a transformation $U(R)$ [or a series of transformations in stages, for the manyelectron case], and a sharply truncated set of adiabatic states is obtained for use in a collision problem.

However, the original basis set is either fully diabatic (class $F$ ), or substantially so, if class $V$ states are used; in any case, the basis states are single-center states and there is no doubt about the correct form for ETF's and the resulting matrices $A_{F}^{R}$ and $\gamma_{F}^{R}$. If the matrix $U(R)$ is available, we may then transform the matrices $P^{R}, A_{F}^{R}$, and $\gamma_{F}^{R}$ according to Eqs. (III. 29) and (III. 30) and then truncate to the relevant square submatrices corresponding to the adiabatic states considered. Within this truncated subspace, we may now identify in a piecewise manner each of the couplings ( $h$ is diagonal):

(i) $P_{F}^{R^{\prime}}=U^{\dagger} P_{F}^{R} U$ : that part of $P^{R}$ which represents only displacement;

(ii) $A_{F}^{R^{\prime}}=U^{\dagger} A_{F}^{R} U$ : this arises from the ETF in the original basis, and exactly cancels $P_{F}^{R^{\prime}}$;

(iii) $P_{\xi}^{R^{\prime}}=U^{\dagger} P_{\xi}^{R} U$; that part of $P^{R}$ which arises from the slow variations with $R$ of the parameters in the class $V$ functions;

(iv) $P_{C}^{R^{\prime}}=-i \hbar U^{\dagger} S^{-1}(d U / d R)$ : the nonadiabatic couplings which arise from the (sometimes rapid) changes in the coefficients of transformation;

(v) $\gamma_{F}^{R^{\prime}}=U^{\dagger} \gamma_{F}^{R} U$ : last, but not necessarily least or negligible.

Obviously, only the last three pieces need actually be calculated. The great advantage of this approach is that 
it clearly allows us to identify, within the limits of the original basis set, all the real nonadiabatic couplings in the problem; and to attribute each to a clearly distinguishable source. The transformation to a suitable truncated diabatic representation can then be carried out by a matrix $W$ which can be defined in several ways; in any case, it satisfies a differential equation of the form

$$
i \hbar(d W / d R)=D W \text {. }
$$

We may take any one of the following:

(i) $D=P_{C}^{R^{\prime}}$; in most cases, this is the significant choice: It eliminates the rapid variations arising from changing coefficients, leaving only the smaller velocity couplings due to slow variations in the parameters $\zeta$ and to the Greek stuff $\gamma_{F}^{R^{\prime}}$. If this were applied to the full (untruncated) space, it would just take us back to the original class $V$ basis. If the construction of the adiabatic representation were done in successive transformations (e.g., SCF-LCAO-MO calculation followed by configuration mixing), then $P_{C}^{R^{\prime}}$ will have separate pieces arising from each stage and the most significant of these may be retained while ignoring the rest.

(ii) $D=P_{C}^{R^{\bullet}}+P_{\mathrm{b}}^{R^{\bullet}}$; this also eliminates the couplings which arise from slow parameter variations. The resulting diabatic states will therefore be closer to class $F$ than the original basis; in the limit of a complete set of states, a fully $F$-diabatic representation will be obtained.

(iii) $D=P_{c}^{R^{\prime}}+P_{\xi}^{R^{\prime}}+\gamma^{R^{\prime}}$; this eliminates all velocitydependent couplings from the new representation, which is therefore $M$ diabatic. In general, the new states are still molecular, but they will be "slowly varying" in the sense that counts. Note however that in general $D^{\dagger} \neq D$ and the transformation is not unitary, so Eq. (IV. 7) becomes

$$
i \hbar d / d t d^{\prime \prime}=\left(S^{-1}\right)^{\prime \prime} h^{\prime \prime} d^{\prime \prime} .
$$

We may remark here that the physical description of fered by computing couplings from the original basis states is equivalent to that obtained if we compute $A_{M}^{R}$ in the adiabatic representation directly, using the special formula (III. 33) to define our class $M$ ETF's; but here we have analyzed it in a piecewise manner.

Direct synthesis of a practical $A_{M}^{R}$ in adiabatic representation. It may happen that the information necessary to obtain the separate pieces of the nonadiabatic couplings as above is not readily available, or it may be that the labor required to do so is not justified by the approximations inherent in the collision problem considered. Then it is a reasonable procedure to introduce a suitable switching function directly into the adiabatic representation and calculate the matrix $A_{M}^{R}$ (we still require $P^{R^{\prime}}$, of course), and find the $M$-diabatic representation obtained by solving Eq. (IV. 8) with $D=P^{R^{\prime}}+A_{M}^{R}$. We have earlier discussed the particularly simple result that appears if a single switching function is used, i.e., the diabatic states are orthonormal.

In performing this task, one should keep in mind the approximate nature of one's objectives. For example, in many cases, it may be possible to estimate the ETF correction effects rather than compute them explicitly, and then just let $D$ be the remaining "large" part of $P^{R}$ which one wishes to eliminate. The test of success then lies in the ability to arrive at a diabatic description with a reasonably accurate account of the matrix $h$ in Eqs. (IV. 7).

Construction of $F$-diabatic states from a given class $M$ representation. Consider now the following problem. Suppose we are handed an arbitrary finite set of class $M$ states, and we wish to construct from these states a set that is $F$-diabatic within the finite manifold. To accomplish this, we must evaluate the matrices $P^{R}$ and $p^{R}$ within the finite manifold, and transform by a matrix $X$ such that

$$
P^{R^{\prime}}+p^{R^{\prime}} S^{-1{ }^{\prime}} \kappa^{\prime}=0
$$

with $S^{-1 *} \kappa^{\prime}$ a diagonal, $R$-independent matrix. It follows immediately from the transformation rules that $X$ must satisfy the differential equation

$$
i \hbar S d / d R X=P^{R} X+p^{R} X S^{-1}{ }^{\prime} \kappa^{\prime},
$$

In most cases, the original representation will have the property that each state correlates to a unique class $F$ state as $R-\infty$, and then in that limit $X(R) \rightarrow 1$. There will then be no ambiguity about $\kappa^{\prime}$ in that limit, and since $S^{-1^{\prime}} \kappa^{\prime}$ must be independent of $R, \mathrm{Eq}$. (IV. 10) is completely specified, and integration will give the matrix transforming to the $F$-diabatic representation. ${ }^{29}$

Unless the original class $M$ representation is complete, the resulting states will not be class $F$, but they will be "close to" class $F$ in the sense that $(\partial / \partial R$ $\left.+\kappa_{n} \partial / \partial r\right) \phi_{n}^{\prime}(r ; R)$ will be orthogonal to all states in the manifold (of course, in a small manifold, they may not look at all like class $F$ states).

It is significant that in this process the ETF's need not be specified in advance. Instead, we can calculate the special $A_{F}^{R}$ matrix for the original class $M$ basis from the transformation matrix $X$ as

$$
A_{F}^{R}=p^{R} X^{t-1} S^{-1}{ }^{\prime} \kappa^{\prime} X^{-1} \text {. }
$$

Partition of the Hamiltonian. An important conclusion of this paper is that many of the intuitive procedures used to construct diabatic representations in the past are formally justifiable. We can show this as follows:

Suppose the Hamiltonian can be partitioned in some way, $h=h_{0}+h_{1}$, and we begin in a representation where $h_{0}$ (a major part of $h$ ) is diagonal, and $h_{1}$ is off-diagonal but small. We may say that such a representation is adiabatic with respect to $h_{0}$, but diabatic with respect to $h_{1}$. Let us now show that this language is completely consistent with our previous definitions of diabatic states. In this representation, the coupled equations are

$$
i \hbar d / d t d=\left[h_{0}+h_{1}+v^{R}\left(P^{R}+A^{R}\right)+v^{R} \gamma^{R}\right] d .
$$

We have included $\gamma^{R}$ here because the representation is not fully adiabatic, but now recall the argument made earlier: $\gamma^{R}$ is proportioned both to $v^{R}$ and to $h_{1}$ and can be neglected in comparison with the terms $h_{1}$ or the terms $v^{R}\left(P^{R}+A^{R}\right)$. The coupled equations then become 


$$
i \hbar d / d t d=\left[h_{0}+h_{1}+v^{R}\left(P^{R}+A^{R}\right)\right] d .
$$

Now, if (and only if) the velocity couplings in Eq. (IV. 11b) are small enough to be considered negligible, then these equations are equivalent to Eqs. (IV. 7) and this representation is obviously $M$ diabatic in the approximate sense required. Let $U(R)$ be the further transformation to the fully adiabatic representation; the transformed equations are then

$$
\begin{aligned}
i \hbar d / d t d^{\prime}= & {\left[\left(h_{0}^{\prime}+h_{1}^{\prime}\right)+v^{R}\left\{U^{\dagger}\left(P^{R}+A^{R}\right) U\right\}\right.} \\
& \left.-i \hbar v^{R} U^{\dagger}(d U / d R)\right] d^{\prime} .
\end{aligned}
$$

Should we find that the velocity-dependent couplings in Eq. (IV.12) are out of hand, (i) we can be sure that the source of the trouble is the $d U / d R$ term and (ii) the easy way to get to the diabatic representation required is not to integrate Eqs. (IV. 8) but to refrain from the damaging transformation $U(R)$. Practically all useful diabatic representations have been constructed by intelligent behavior of this kind, based on some relevant partition of $h$.

\section{Many-electron systems}

All conclusions of this and the preceding Sec. III are generally valid for many-electron systems also, provided we give definitions for the ETF and for the matrices $\rho, \sigma, A, \gamma$, etc. which it generates. Since the remaining discussion in this paper is general in its intent too, it is appropriate at this point to indicate briefly how such ETF's and matrices may be defined.

Class $F$ states. A class $F$ state of an $N$-electron diatomic system is an antisymmetrized product of class $F$ atomic substates for each of the atomic subsystems $\mathrm{A}$ and $\mathrm{B}$ :

$$
\Psi=\mathscr{A}_{N} \Psi_{n_{\mathrm{A}}}^{\mathrm{A}} \Psi_{n_{\mathrm{B}}}^{\mathrm{B}},
$$

where $A_{N}$ is the antisymmetrizer. $\Psi_{n_{A}}^{A}$ describes a class $F$ atomic substate for $N_{\mathrm{A}}$ electrons $\left(N_{\mathrm{A}}+N_{\mathrm{B}}=N\right)$, including spin, which we do not indicate explicitly; its important property is that it depends on the spatial coordinates of the $N_{\mathrm{A}}$ electrons in a homogeneous way, i. e.,

$$
\begin{aligned}
& \Psi_{n_{\mathrm{A}}}^{\mathrm{A}}\left(\mathbf{r}_{1}, \mathbf{r}_{2}, \ldots, \mathbf{r}_{N_{\mathrm{A}}} ; R\right)=\Psi_{n_{\mathrm{A}}}^{\mathrm{A}}\left(x_{1}, y_{1}, z_{1}-\kappa_{\mathrm{A}} R ; x_{2}, y_{2},\right. \\
& \left.\quad \times z_{2}-\kappa_{\mathrm{A}} R ; \ldots ; x_{N_{\mathrm{A}}}, y_{N_{\mathrm{A}}}, z_{n_{\mathrm{A}}}-\kappa_{\mathrm{A}} R\right) .
\end{aligned}
$$

There is therefore no ambiguity about the appropriate ETF

$$
\begin{aligned}
F_{n_{\mathrm{A}}}^{\mathrm{A}}= & \exp \left\{(i m / \hbar) \mathbf{v} \cdot\left[\frac{1}{2} \kappa_{\mathrm{A}}\left(\rho_{1}+\rho_{2}+\cdots+\rho_{N_{\mathrm{A}}}\right)\right]\right. \\
& \left.-(i m / 2 \hbar) N_{\mathrm{A}} \kappa_{\mathrm{A}}^{2} \int^{t} \mathbf{v}^{2} d t^{\prime}\right\} .
\end{aligned}
$$

Since this ETF is a symmetric function of the electron coordinates, its presence does not affect the permutation symmetry of the product $F_{n_{A}}^{A} \Psi_{n_{A}}^{A}$; and the further antisymmetrizing of the joint products of the $A$ and $B$ states produces a class $F$ scattering state.

Class $F$ states represent the actual limiting electronic wave function of the system as $R \rightarrow \infty$, and in principle any state at any internuclear distance could be represented as a linear combination of such states with their appropriate ETF's attached. In practice, however, such representations require a very large number of terms, and are of questionable utility.

Molecular states and orbital products. A more practical way of obtaining a many-electron ETF and the corresponding matrices $A$ and $\gamma$ is provided by a different approach. Usually, a molecular wave function is represented as a linear combination of orbital product basis functions, i.e., antisymmetrized products of one-electron orbitals (including spin), one for each of the $N$ electrons in the system. (This statement holds not only for molecular orbital products, based on a self-consistent-field problem of some kind, but also for valencebond-type wave functions.)

To each one-electron orbital in an orbital product, we may associate a switching function appropriate to that orbital (if the orbital is itself of class $F$, i.e., atomic, we may of course take $\frac{1}{2} f_{n}=\kappa_{\mathrm{A}}$ or $\kappa_{\mathrm{B}}$ ) and the ETF for the product basis state is just a product of the resulting ETF's for each orbital. The ETF-modified basis states are then just antisymmetrized products of such an ETF and the corresponding orbital product.

The important point in such a procedure is that the role played by each orbital switching function is linked to the role of the corresponding orbital in one-to-one fashion. There are well-known theorems regarding the matrix elements of one- and two-electron operators between orbital product basis states, and the operators which define all the matrices $(\rho, \sigma, A, \gamma$, etc. $)$ of interest to us have the formal properties of one-electron operators. The essential conclusion is that for an orbital product both $\mathrm{P}$ and $\mathrm{A}$ consist of a sum of contributions for each orbital.

Simplifications. The above description shows how we can go about a really correct calculation: Construct the appropriate ETF's, compute the resulting matrices $A, \gamma$, etc., for orbital product states (or class $F$ states), and transform to a suitable representation, possibly the adiabatic one which makes $h$ diagonal, in the usual way. Then, if desired, a truncation to a limited manifold of adiabatic states may be made, and from there the definitions and methods given above are all applicable. Fortunately, however, good results can usually be obtained more simply. We still need to know the matrix $\mathbf{P}$ (in adiabatic representation) but instead of calculating the ETF corrections, we may (sometimes merely by inspection) delete from $\mathbf{P}$ that part which corresponds simply to displacement; then what remains of $\mathbf{P}$ after such "background correction" corresponds to real nonadiabatic coupling. To transform to a diabatic representation, we may either diagonalize this coupling by solving Eq. (IV. 8), or, if an obviously related partition of the Hamiltonian is apparent, undo the transformation which made the relevant $h_{1}$ diagonal.

\section{Spin-orbit couplings}

In many collision processes, the spin-orbit effects play a negligible role, and they need not be included in the electronic Hamiltonian. When they are important, they are usually sufficiently small that they are pref- 
erably left off diagonal. In typical calculations, ${ }^{30} \mathrm{a}$ convenient representation is obtained by diagonalizing only the electrostatic part of $h$; such a representation is diabatic with respect to spin-orbit couplings, but adiabatic with respect to electros tatic effects. The states of different electrostatic symmetry that are connected by spin-orbit couplings are not connected by $P+A$, no matter how $A$ is defined. Thus, the formal definition is in accord with this terminology.

At present, there is no universally accepted nomenclature for representations in systems of this type. We propose the following definitions, which are both historically and physically accurate: Let us say that the Born-Oppenheimer representation is the one for which only the electrostatic parts of $h$ are diagonalized; then, for example, for crossings in which the states are connected only by spin-orbit couplings, the Born-Oppenheimer states behave diabatically. We will say that the adiabatic representation is the one in which the entire $h$ is made diagonal; in the above case, unlike the BornOppenheimer eigenvalues, the adiabatic eigenvalues will avoid crossing.

\section{Angular couplings}

In cases where angular couplings are important, the best approach to the problem of diabatic and adiabatic states is as follows: The angular coupling term is $v^{\Theta}\left(-L_{y} / R+A^{\ominus}\right)$ and since the collision angular momentum is conserved (in the classical trajectory approximation), we can write $R v^{\ominus}=b v^{0}$, where $b$ is the collision impact parameter and $v^{0}$ the limiting collision speed. Then this coupling plays the role of a potential, i.e., we can regard it as just another part of the effective Hamiltonian (even though it depends on $b$ and $v^{0}$ ) and ignore its origin as a dynamic effect. This interpretation is fully consistent with the implications of the terms diabatic/adiabatic in the high-and low-velocity limits.

Thus, for example, we would say in particular that for slow collisions, the Born-Oppenheimer representation is the diabatic representation in which only the electrostatic part of the Hamiltonian is made diagonal, while the angular coupling $\left(v^{0} b / R\right)\left[-L_{y} / R+A^{\theta}\right]$ is off diagonal and can cause transitions to states of different diatomic symmetry [such states are not coupled by $v^{R}\left(P^{R}+A^{R}\right)$ ]. Correspondingly, the adiabatic representation is the one in which the entire effective potential

$$
V=h+\left(v^{0} b / R\right)\left[-L_{\mathrm{y}} / R+A^{\Theta}\right]
$$

is made diagonal at each $R .^{31}$ In this representation, all transitions among states are produced by the coupling $-i \hbar v^{R} U^{\dagger}(R) d U / d R$, where $U(R)$ is the transformation from the Born-Oppenheimer representation to this adiabatic representation. Estimates of typical sizes of the relevant couplings and application of the rules we will present later lead to the conclusion that this adiabatic representation is rarely useful in practice, the BornOppenheimer one being normally the appropriate one for slow collisions.

Evidently, a new situation arises either in the case of high collision velocity $v^{0}$, or in the case of coupling at very large $R$, since then the angular couplings may be much stronger than the interactions coupling the electronic system to the internuclear axis. In such a case, we are led to consider another sort of (diabatic) representation in which $\left(-L_{y} / R+A^{\Theta}\right)$ is diagonal, while the couplings to the internuclear axis in the Born-Oppenheimer Hamiltonian are off diagonal. It is easy to show that the basis functions of this representation are nonrotating orbitals. It is obvious that such orbitals are indeed suitable for fast collisions, and such a description was used by Shakeshaft ${ }^{26}$ to study $\mathrm{H}^{*}-\mathrm{H}$ collisions at energies of $25-200 \mathrm{keV}$.

However, even for slow collisions, there is a domain at very large $R$ where the angular coupling interaction becomes stronger than the axial couplings in $h$. In a collision, the system must somehow pass between the situation at finite $R$, where the axis coupling effects in $h$ are large (hence, where $h$ should be made diagonal and angular coupling is off diagonal and small), and the situation at $R-\infty$, where the reverse is true. Formally, this would lead to a complicated dynamical problem involving coupling within degenerate manifolds of a given electronic angular momentum; such a coupling problem would exist at the beginning and end of every collision, no matter how slow. ${ }^{32}$ Fortunately, in the overwhelming majority of cases, that problem has an essentially trivial and physically obvious solution. ${ }^{33}$

To understand the situation, it is most helpful to use the terminology introduced in the Appendix to discuss a fundamentally similar problem, the case of "near-symmetric resonance" first discussed by Rosen and Zener. ${ }^{34}$ The essential point is that two distinct diabatic representations must be considered. For this case, the $i n-$ ternal diabatic representation is the one in which $h$ is diagonal and the angular coupling $\left(L_{y} / R+A^{\Theta}\right)$ is off diagonal, and the external diabatic representation is the one in which the angular coupling is diagonal and the axial couplings in $h$ are off diagonal. In the internal diabatic representation, the (Born-Oppenheimer) basis states rotate with the internuclear axis; in the external diabatic representation, the basis states are nonrotating. As discussed above, we can also define the adiabatic representation as that which makes the entire potential $V$ diagonal [Eq. (IV. 16)].

The problem then is how to connect actual states of the system as it passes back and forth between internal and external regions. It can be shown that, in virtually every case, the correct solution of the dynamical problem is as follows: Find the (large) radius $\hat{R}$ at which the electrostatic splitting contained in $h$ is equal in magnitude to the angular coupling $\left(v^{0} b / R\right)\left(-L_{y} / R+A^{\theta}\right)$ between the same pair of states; at this $\hat{R}$, make the sudden connection between the Born-Oppenheimer states (labeled by $\Lambda$ ) and the asymptotic atomic states (labeled by $\left.L_{\mathrm{A}} M_{\mathrm{A}} L_{\mathrm{B}} M_{\mathrm{B}}\right)$. Normally, $\hat{R}$ is so large that it can be considered to be infinite for practical purposes. This sudden connection is consistent with the rules given in Sec. V.

For special cases where the axial couplings never dominate over angular couplings (e.g., Rydberg states), or for cases where both spin-orbit interaction and angu- 
lar coupling must be considered in the external region, the situation can become much more complicated; transitions among the Hund's coupling cases ${ }^{35}$ must be considered and in fact a case of no spectroscopic interest, case (e), always arises. A discussion of the problem in this more complicated situation has been given by Mies $^{36}$ and applications of similar ideas to some particular examples have been made by Tolk et al. ${ }^{37}$ Again the problem may be discussed using the rules we give in Sec. $V$ and the above definitions of diabatic and adiabatic states.

\section{E. Generalization to arbitrary velocity}

In deriving Eqs. (III. 12), we made approximations which are appropriate only for slow collisions, namely, the neglect of terms of order $v^{2}$ and higher in the expansion of the ETF products $F_{k}^{*} F_{n}$ in Eqs. (III. 4). This permitted us to define the problem in terms of electronic basis states and matrix elements which are independent of velocity. However, these approximations need not have been made. Equations (III.4) are valid if we neglect terms of order $(d \mathrm{v} / d t)$ and some terms proportional to $v^{2} \nabla_{R} f$ and $v^{2}\left(f^{2}-1\right)$, and if we use a class $F$ basis and the corresponding single center ETF's, the $v^{2}$ terms vanish exactly. This means that we could have developed the theory of diabatic states in an analogous way to that given here, provided we used the matrices $S(\mathrm{v}), h(\mathrm{v})$, $\mathbf{P}(\mathbf{v})+\mathbf{A}(\mathbf{v})$, etc., instead of their zero-velocity limits, and the general statements about diabatic states then would not depend on a low-velocity approximation. In particular, class $F$ states are again the basis for a fully $F$-diabatic representation-and if we take all vector components of $\mathbf{P}(\mathbf{v})+\mathbf{A}(\mathrm{v})$ into account, these class $F$ states would also be nonrotating. This allows us to make contact with an atomic state representation as a fully diabatic description, i. e., the description which is most appropriate for fast collisions. This is mainly of formal interest, though, because the problem of diabatic vs adiabatic states has been of practical interest only for slow collisions.

\section{OPTIMAL REPRESENTATIONS}

We have given a formal definition of adiabatic and diabatic states, and we have pointed out that the useful representations for slow collisions will normally be adiabatic with respect to certain couplings and diabatic with respect to others. As the considerations of the preceding section suggest, there are two ways to construct such representations. Starting from an adiabatic representation, a diabatic representation can be obtained by calculating the matrix $W(R)$ satisfying Eq. (IV. 8), so that the desired part of the new $P^{R}+A^{R}$ vanishes. (This method will normally be useful only if the adiabatic representation has been truncated to a very few states.) On the other hand, if a class $F$ basis is the starting point, the representation is already fully diabatic, and its diabatic character can be partially retained by selective partial diagonalization of $h$ \{or, more generally, $V$ [cf. Eq. (IV. 16)]\}.

Most often, however, the starting point of a collision calculation is a class $V$ basis, consisting of one-center functions with variable orbital exponents or other parameters. Such a representation is neither adiabatic nor diabatic, but mixed: A general pair $(j, k)$ of states will be coupled by both $\left(P^{R}+A^{R}\right)_{j k}$ and $V_{j k}$. However, since the orbital exponents will usually be slowly varying functions of $R$, it will frequently happen that $v^{R}\left(P^{R}+A^{R}\right)_{j k}$ will be substantially smaller than $V_{j k}$. When this holds, we can say that the class $V$ basis is approximately diabatic, and it can be treated as a diabatic representation for all practical purposes.

The above ideas suggest that it may be appropriate to re-examine a very old question: of all the possible representations of a given collision system, can we pick the "optimal" one, in which the coupling between the states is as weak as possible? Although it is unlikely that a completely general answer to this question will ever be found, a cautiously affirmative answer can be given if the question is suitably restricted.

We consider only the partially diabatic and partially adiabatic representations, in which the coupling between a pair of states is represented either by $V_{j k}$ or $P_{j k}^{R}+A_{j k}^{R}$. Even with this restriction, three more limitations are evident. First, the optimal representation, if it exists at all, necessarily depends on the collision velocity. Second, for many collision processes, there is a range of intermediate velocities for which the system shows an intrinsic strong coupling, and for which there is no representation of the type considered here in which the coupling is weak. The standard Landau-Zener model displays both of these points quite clearly: At low velocities, the coupling is weakest in the adiabatic representation; at high velocities, it is weakest in diabatic representation; but at intermediate velocities, the states are strongly coupled, and the usual kinds of changes of representation do not help.

A final limitation is also apparent. It is only possible to give general rules for the construction of optimal representations if there are general models that at least approximately describe the behavior of a wide variety of systems. For curve crossings, the Landau-Zener model has such broad applicability, and it has often been used to estimate the diabaticity or adiabaticity of a given collision. For states that are strongly coupled but do not cross, an appropriate model is one first suggested by Rosen and Zener and later modified and extended by a number of workers. ${ }^{34}$ The results of this model and its application to collisional transitions are summarized in the Appendix. Now, since these standard models are based upon an orthogonal diabatic representation, we have to assume that such a representation can be constructed..$^{38}$

Within these limitations and restrictions, a simple prescription can be given for choosing a good representation. It must be recognized that we are asking quite a modest question: For each pair of states $(j, k)$ in the system, is it better that the coupling between them be represented diabatically, by $V_{j k}$, with $\left(P^{R}+A^{R}\right)_{j k}=0$, or adiabatically, by $\left(P^{R}+A^{R}\right)_{1 k}$, with $V_{j k}=0$ ? For a system with $N$ states, we then have $\frac{1}{2} N(N-1)$ choices. This set of choices can normally be made by a sequence of 
RULES FOR OBTAINING A USEFUL PARTIALLY DIABATIC REPRESENTATION

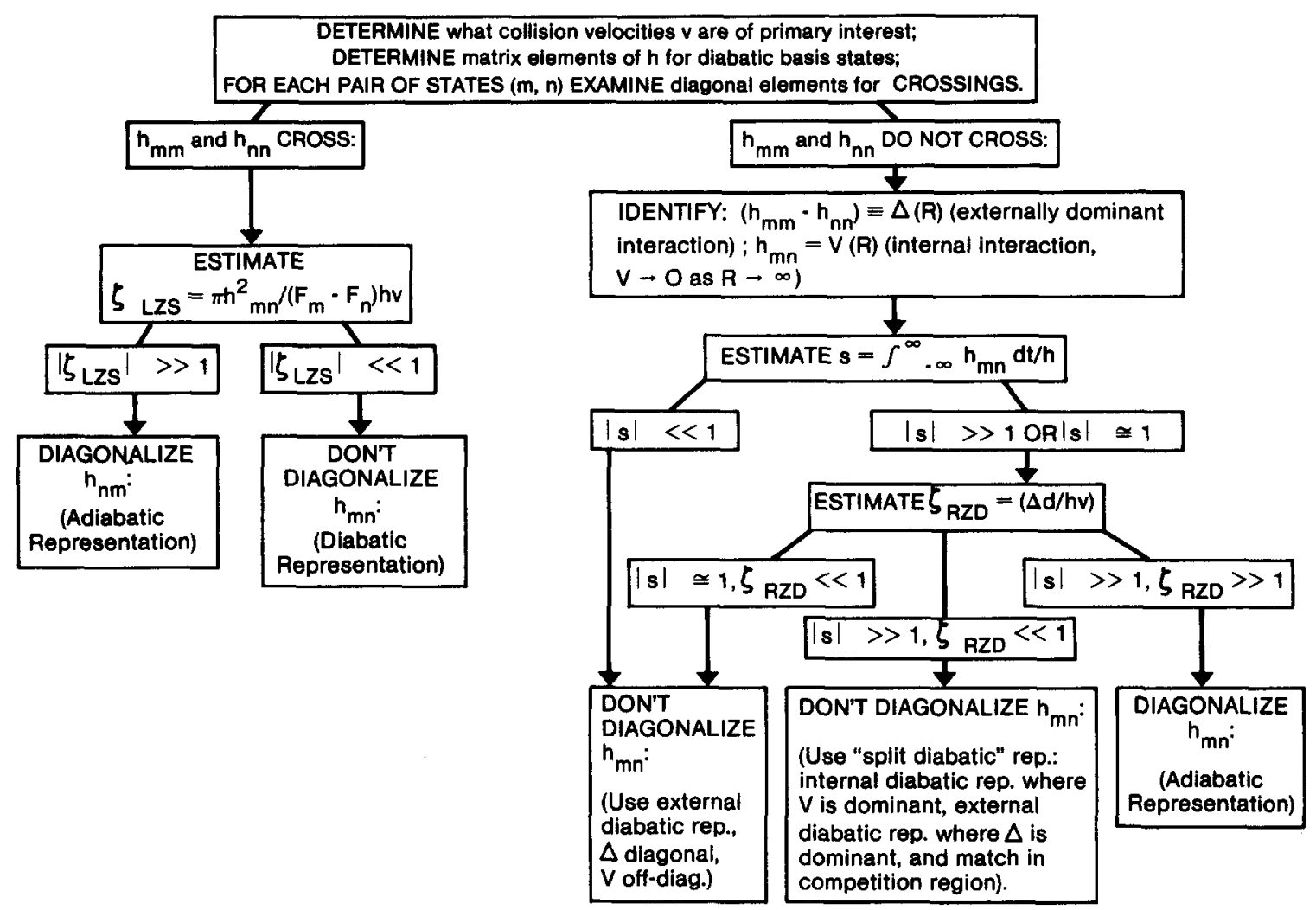

FIG. 1. Rules for obtaining a useful partially diabatic representation.

binary decisions, each decision involving only one pair of states. Although one decision may influence another, a self-consistent set of choices can usually be reached by beginning with the most strongly coupled pairs.

The procedure for making the binary decisions can be condensed into a set of rules or guidelines. As stated here, the rules assume that the starting representation is diabatic between the given pair of states under consideration, so we are deciding whether to diagonalize $V_{j k}$ or leave it off-diagonal. The steps in the procedure are as follows:

(1) Decide what collision velocity is of primary interest. The optimal representation necessarily depends on the collision velocity.

(2) Examine the curves $V_{j j}(R)$ and $V_{k k}(R)$ for crossings. If they cross, apply rule (3); if not, apply rules (4) and (5).

(3) For crossing states, estimate the Landau-Zener parameter

$$
\delta_{\mathrm{LZS}}=2 \pi V_{j k}^{2} / \hbar v\left|F_{j j}-F_{k k}\right|,
$$

where $F_{j g}$ is the force $-\left(d V_{j j} / d R\right)$. If $\delta_{\mathrm{Lzs}} \gg 0.69$, diagonalize $V_{j k}$ to represent this coupling adiabatically; if $\delta_{\text {Lzs }} \ll 0.69$, do not diagonalize $V_{j k}$.

(4) For noncrossing states (cf. the discussion of the Rosen-Zener-Demkov problem in the Appendix); estimate the two parameters

$$
s_{\infty}=\int_{R_{0}}^{\infty}\left[V_{j k}(R) / \hbar v(R)\right] d R
$$

and

$$
\zeta_{\mathrm{RZD}}=\Delta d / \hbar v,
$$

where, as in the Appendix, $d$ is the distance over which $\Delta(R)=\left|V_{j j}(R)-V_{k k}(R)\right|$ is comparable to $V_{j k}(R)$, and $\Delta$ is the average of $\Delta(R)$ over that region.

(5) (a) if $s_{\infty} \ll 1$ or if $s_{\infty} \simeq 1$ and $\zeta_{\mathrm{RZD}} \ll 1$, do not diagonalize $V_{j k}$; use the external diabatic representation. (b) If $s_{\infty} \gg 1$ and $\zeta_{\mathrm{RzD}} \gg 1$, diagonalize the $V$ matrix to use the adiabatic representation. (c) If $s_{\infty} \gg 1$ and $\delta_{\mathrm{RZD}} \ll 1$, then the "split" representation may be used if convenient (see Appendix).

(6) In the intermediate cases (as for $\delta_{\mathrm{Lzs}} \simeq 1$ or $s_{\infty} \simeq 1$, $\zeta_{\mathrm{RZD}} \simeq 1$ ), the problem intrinsically involves strong coupling, and the choice of representation probably doesn't matter.

Figure 1 summarizes the above in a simple flow chart.

Although these rules have not been explicitly written down before as part of a procedure for constructing an optimal representation, they are not really new either: for example, the Landau-Zener formula has been used for many years in this way. Note also that, in writing rule (3), we are not assuming that the Landau-Zener formula gives an accurate transition probability-examples of its breakdown are well known; we are only as - 
suming that it can provide the relatively gross distinction between essentially diabatic and essentially adiabatic behavior. Similarly, although the binary decisions are necessarily based on two-state models, we are not assuming that any two state truncation gives an adequate description of the given system, nor are we even assuming that the collision can be described by a sequence of isolated two-state interactions. In the above rules, the models are used only to choose a good representation, one in which an accurate solution may conveniently be obtained.

\section{SUMMARY}

Starting from the classical trajectory formulation and making approximations suitable for slow collisions, we have arrived at sets of coupled equations (III. 12) which include the effects of electron-translation factors (ETF's). Such equations can be derived either using single-center class $F$ or class $V$ basis states and the corresponding single-center ETF's, or using two-center class $M$ (molecular) basis states and ETF's based on switching functions. In these equations, the matrices $S, h$, and $\mathbf{P}$ are the same as those which would appear in a formulation ignoring ETF's (e.g., perturbed-stationary-states theory). The matrix $A$ arises from the action of differential operators on the ETF and is especially important in the formal theory, since it identifies and removes the part of $\mathbf{P}$ that represents translation of the basis states with the moving nuclei; the corrected coupling $(\mathbf{P}+\mathbf{A})$ represents actual deformation or change of character of the states. In addition, there are terms, collectively denoted $\gamma$, which arise from the expansion of ETF products $F_{k}^{*} F_{n}$ in powers of $\mathrm{v}$. These terms vanish if the basis states are eigenfunctions of $h$, or if a common switching function is used for all the states (in either case the basis is class $M$ ); otherwise, $\gamma$ cannot be shown to be negligible.

Although the form of Eqs. (III. 12) is preserved under basis transformations of the usual kind, the couplings obtained by starting with class $F$ or class $V$ basis states, using single-center ETF's, and then transforming to a class $M$ basis are not necessarily the same as those obtained directly in the class $M$ basis, using ETF's with switching functions. If we require that they be the same, we obtain a unique specification of the $M$-basis switching functions via the transformation linking the class $V$ or $F$ basis to the $M$ basis. This formula is of some interest for the problem of choosing good switching functions.

We defined adiabatic states in completely conventional ways, except that when angular coupling or spin-orbit coupling is involved there is no uniformly used convention; we have defined the adiabatic representation in such cases as that in which the entire effective Hamiltonian $V$ is diagonal. We use the name Born-Oppenheimer representation to describe that in which only the usual electrostatic part of $h$ is diagonal, and which therefore is diabatic with respect to the remaining couplings.

We defined diabatic representations as those in which all or a selected portion of the matrix $P+A$ vanishes; following common usage, we may also say that a repre- sentation is diabatic if the relevant parts of $P+A$ are negligible. Two distinct types of radial diabatic representation then appear, depending on how $A$ is defined.

Class $F$ states, which translate with the nuclei to which they are attached and which rotate with the internuclear axis, but do not change in any other way form a basis which is fully $F$ diabatic $P^{R}+A_{F}^{R}=0$. In this representation, the velocity-dependent couplings do not generally vanish, since $v \cdot \gamma \neq 0$.

$M$-diabatic representations, for which $P^{R}+A_{M}^{R}=0$, fulfill the condition that the velocity-dependent couplings vanish. In general, the corresponding basis states must change to some extent as $R$ changes, and are not class $F$ states; in many cases, they have molecular character. However, for most purposes, what matters is the form of the diabatic coupled equations, not the basis states; in that sense, $M$-diabatic representations provide the formal basis for the traditional paradigm of a diabatic state.

For slow collisions, most useful representations are partially diabatic and partially adiabatic; most often, it is convenient to make matrix elements of $P^{R}+A^{R}$ vanish only within a small manifold of strongly coupled states. The most convenient representation might then be diabatic for transitions within this small manifold, but adiabatic with respect to transitions outside this manifold.

In many problems, the magnitude of $A$ (and of the part of $P$ which it corrects) is negligible compared to the couplings of interest. For example, in systems having a curve crossing at small or moderate internuclear distances, estimated typical values of $A^{R}$ are much smaller than the values of $P^{R}$. For such systems, then, good approximately diabatic states can be constructed by making $P^{R}$ vanish, as Smith ${ }^{10}$ originally suggested. Whenever the relevant matrix elements of $A^{R}$ are negligible, then provided that the diabatizing transformation is carried out only within a small manifold, the seemingly severe formal defect in Smith's approach is simply unimportant. ${ }^{39}$ However, the formal definitions and methods described here show how the corrections for translation can be made whenever their effects are not negligible.

Having obtained a formal definition of diabatic states, and of partially diabatic and partially adiabatic representations, it was natural to re-examine the very old question of whether there is a representation of the above type that is particularly convenient. Here we condensed physical intuition and a lot of experience by many workers into a set of guidelines that are easily applied and which should normally lead to a good representation. Of course, changing the representation does not solve the problem. It may, however, provide additional physical insight, suggest approximations, or simplify numerical integration.

Finally, in view of the simple and almost obvious character of most results of this paper, it may be asked, Why has a general definition of diabatic states been so elusive? To answer this, we must first emphasize that there has never been any great difficulty in actually constructing diabatic states; through physical intuition and 
insight, good diabatic representations have been found for a great variety of systems. ${ }^{2-9}$ However, the reason that a formal definition could not be found was that there was a flaw in the formalism: Electron translation effects were too often omitted from the theory of slow collisions. In fact, it was only very recently that a way was found to include them in a fully quantum-mechanical description. The essential result of this paper is that the ETF's and the resulting couplings repair the defect in the formalism, and thereby make possible a general definition of diabatic states which is in accord with the intuitive approaches that have been used in the past.

\section{ACKNOWLEDGMENTS}

This research was supported in part by Research Corporation, the National Science Foundation, and the National Research Council of Canada.

\section{APPENDIX: QUALITATIVE BEHAVIOR OF TWO- STATE NONCROSSING INTERACTIONS}

When diabatic potential curves are strongly coupled but do not cross, there is a somewhat greater variety of possible behavior than appears in the crossing case. The case of accidental asymptotic near degeneracy (also called "perturbed symmetric resonance") is the most complicated of the common situations, and it provides a basic model within which the simpler situations can also be understood.

We define

$$
\Delta(R)=V_{22}(R)-V_{11}(R)
$$

and we assume that $\Delta(R)$ is positive and slowly varying for all $R$. We assume that the states are coupled by an interaction represented by $V_{12}(R)$ such that $V_{12}(R) \ll \Delta(R)$ for large $R$ but $V_{12}(R) \gg \Delta(R)$ for small $R$. We assume that the representation is properly formulated so that as $R \rightarrow \infty, V_{12}-0$ but $\Delta(R)-$ constant.

The adiabatic representation is as usual the one in which the $2 \times 2 \mathrm{~V}$ matrix is everywhere diagonal. Two distinct diabatic representations are of interest: the "external" diabatic representation (the usual one) in which $\Delta(R)$ is diagonal and $V_{12}(R)$ is off diagonal, and an "internal" diabatic representation, which is transformed such that the original $V_{12}(R)$ is put onto the diagonal and $\Delta(R)$ is made off diagonal. The matrix effecting this transformation is

$$
U=2^{-1 / 2}\left[\begin{array}{cc}
1 & -1 \\
1 & 1
\end{array}\right]
$$

i. e. ,

$$
U\left[\begin{array}{cc}
-\frac{1}{2} \Delta(R) & V_{12}(R) \\
V_{12}(R) & \frac{1}{2} \Delta(R)
\end{array}\right] U^{\dagger}=\left[\begin{array}{cc}
-V_{12}(R) & -\frac{1}{2} \Delta(R) \\
-\frac{1}{2} \Delta(R) & V_{12}(R)
\end{array}\right] .
$$

Systems of this type can be described by a model of Rosen and Zener, ${ }^{34(2)}$ which was later modified by Demkov, ${ }^{34(c)}$ and put into the most general possible form by Dinterman and Delos. ${ }^{9(b)}$ Defining the independent variable

$$
s=\int_{0}^{t} V_{12}\left(t^{\prime}\right) d t^{\prime},
$$

the model consists in the assumption that if

$$
T(R)=\Delta(R) / 2 V_{12}(R)
$$

is regarded as a function of the variable $s$, then $T(s)$ can be approximated by the form

$$
T(s) \simeq(\alpha \cos \zeta s)^{-1},
$$

where $\alpha$ and $\zeta$ are constants. Various ways of fitting actual potential curves to the model suggest that two parameters of the potential matrix are particularly important: an integrated coupling strength parameter

$$
s_{\infty}=\int_{0}^{\infty} V_{12}\left(t^{\prime}\right) d t^{\prime}
$$

and an adiabaticity parameter

$$
\zeta_{\mathrm{RZD}}=\Delta \bar{t} / \hbar \text {. }
$$

Here, $\tilde{t}$ is the time during which $V_{12}(R)$ and $\Delta(R)$ are comparable in magnitude (say within a factor of 3 ); this time may be significantly less than the time duration of the entire collision. $\Delta$ is the average of $\Delta(R)$ over this time period $\tilde{t}$.

Now, three types of behavior may occur. In a very fast collision, the system tends to stay in its original precollision state, and so it is most easily described by the external diabatic representation. This situation is obtained if $s_{\infty} \ll 1$. In a very slow collision, if $s_{\infty} \gg 1$ and $\Delta \bar{t} / \hbar \gg 1$, then the system continuousiy adjusts to the change of the $V$ matrix, and the adiabatic representation is most appropriate. At intermediate velocities, however, if $s_{\infty} \gg 1$ and $\Delta \bar{t} / \hbar \ll 1$, then the system may behave adiabatically with respect to $V_{12}$ in the internal region and adiabatically with respect to $\Delta(R)$ in the external region; however, it may pass suddenly from one region to the other. A split representation is suitable for describing this type of behavior: we may use the internal diabatic representation at small $R$ and the external diabatic representation at large $R$, joining the two at some point where $T(R) \approx 1$.

The split representation is normally only useful if there is an asymptotic near degeneracy; in other cases of interaction between noncrossing potential curves, we usually need only to choose between the external diabatic and the adiabatic representations, and the adiabaticity parameter $\zeta_{\mathrm{RZD}}$ is somewhat more important than $s_{\infty}$ in making this choice. Finally, just as in the curvecrossing case, a representation which gives weak coupling can only be found in the limiting cases; otherwise, the problem intrinsically involves strong coupling, and there is no representation of the type we are considering in which the coupling is weak.

${ }^{1}$ C. Zener, Proc. R. Soc. (London) Ser. A 137, 696 (1932). ${ }^{2}$ W. Lichten, Phys, Rev. 131, 229 (1963).

${ }^{3}$ U. Fano and W. Lichten, Phys. Rev, Lett. 14, 627 (1965);

W. Lichten, Phys. Rev. 164, 131 (1967); M. Barat and

W. Lichten, Phys. Rev. A 6, 211 (1972).

${ }^{4} \mathrm{~J}$. Eichler and V. Wille, Phys. Rev. A 11, 1973 (1975);

J. Eichler, V. Wille, B. Fastrup, and K. Taulbjerg, Phys. Rev. A 14, 707 (1976).

${ }^{5}$ (a) V. Sidis and H. Lefebvre-Brion, J. Phys, B 4, 1040 
(1971); (b) M. Aubert and C. LeSech, Phys. Rev. A 13, 632 (1976); (c) B. Andresen and S. E. Nielsen, Mol. Phys. 21, $523(1971)$

${ }^{6}$ R. W. Numrich and D. G. Truhlar, J. Phys. Chem. 79, 2745 (1975); R. S. Berry, J. Chem. Phys. 27, 1288 (1957); J. L. Magee, J. Chem. Phys. 8, 687 (1940); R. Grice and D. R. Herschbach, Mol. Phys. 27, 159 (1974).

${ }^{7}$ M. B. Faist and R. B. Bernstein, J. Chem. Phys. 64, 2971 (1976); M. B. Faist and R. D. Levine, J. Chem. Phys. 64, $2953(1976)$.

${ }^{8}$ J. S. Cohen, Phys. Rev. A 13, 86, 99 (1976).

${ }^{9}$ (a) C. Melius and W. A. Goddard, Phys. Rev. A 10, 1541 (1974); Chem. Phys. Lett. 15, 524 (1972); Phys. Rev. Lett. 29, 975 (1972); (b) T. R. Dinterman and J. B. Delos, Phys. Rev. A 15, 463 (1977).

${ }^{10}$ F. T. Smith, Phys. Rev. 179, 111 (1969).

${ }^{11} \mathrm{H}$. Gabriel and K. Taulbjerg, Phys. Rev. A 10, 741 (1974).

${ }^{12}$ A. Messiah, Quantum Mechanics (North-Holland, Amsterdam, 1965), Vol. II, p. 747.

${ }^{13}$ W. R. Thorson and J. B. Delos, Phys. Rev. A 18, 117 (1978) 18,135 (1978).

${ }^{14}$ W. R. Thorson and H. Levy, II, Phys. Rev. 181, 230, 244, 252 (1969); C. F. Lebeda, W. R. Thorson, and H. Levy, II, Phys. Rev. A 4, 900 (1971); V. SethuRaman, W. R. Thorson, and C. F. Lebeda, Phys. Rev. A 8, 1316 (1973).

${ }^{15} \mathrm{~S}$. K. Knudson, M. Kimura, and W. R. Thorson (in preparation).

${ }^{16}$ J. B. Delos, W. R. Thorson, and S. K. Knudson, Phys. Rev. A 6, 709 (1972); J. B. Delos and W. R. Thorson, Phys. Rev. A 6, 720 (1972).

${ }^{17}$ M. E. Riley, Phys. Rev. A 7, 626 (1973).

${ }^{18}$ It is proven in Refs. 13 that the geometric center, rather than the center of mass of the nuclei, may be taken as the reference origin for the electron coordinate, without affecting the generality of the formalism. A demonstration of this may be given in a fully quantum formulation by noting that if the center of mass of the nuclei be chosen for reference origin, as is normally done, then there is always a term in the ETF which just represents the translation of that center with respect to the geometric center, and which may be formally removed by changing reference origin to the latter. Moreover, it is clear that, at the level of a classical trajectory formulation, the physical description of the system must be invariant to the choice of reference origin for electron coordinates. For another discussion of this point, see A. Riera and A. Salin, J. Phys. B 9, 2877 (1976).

${ }^{19} \mathrm{~A}$ purist might argue that a precise mathematical distinction between class $V$ and class $M$ representations has not been made. Although we prefer to take the view that the classes are easily recognizable in a calculation, a mathematical distinction can be made in the following way, using matrices introduced in later sections: Take an arbitrary representation, and append ETF's suitable to class $V$ states. Then, the representation is strictly class $V$ if and only if the matrix $S^{-1}\left(P+A_{V}\right)$ is block diagonal, such that it connects only states associated with the same $\kappa_{n}$. This definition means that the designation class $V$ is a property of a representation and not of a single state. Class $F$, however, can refer to a single state.

${ }^{20}$ S. B. Schneiderman and A. Russek, Phys. Rev. 181, 311 (1969).

${ }^{21}$ Other forms for $F_{n}$ are also possible, and some are mentioned in Ref. 13.

${ }^{22}$ D. R. Bates, Proc. R. Soc. (London) Ser. A 247, 294 (1958).

${ }^{23}$ D. Rapp, D. Dinwiddie, D. Storm, and T. E. Sharp, Phys. Rev. A 5, 1290 (1972); D. Rapp and D. Dinwiddie, J. Chem. Phys. 57, 4919 (1972); D. Rapp and C. -M. Chang, J. Chem. Phys. 59, 1266, 1276 (1973); D. Rapp, J. Chem. Phys. 58, 2043 (1973); 61, 3777 (1974).

${ }^{24}$ L. Wilets and D. F. Gallaher, Phys. Rev. 147, 13 (1966); 169,139 (1968)

${ }^{25}$ I. M. Cheshire, D. F. Gallaher, and A. Joanna Taylor, J. Phys. B 3, 813 (1970).

${ }^{26} \mathrm{R}$. Shakeshaft, Phys. Rev. A 14, 1626 (1976).

${ }^{27}$ T. A. Green, Proc. Phys. Soc. London 86, 1017 (1965).

${ }^{28} \mathrm{~T}$. F. O'Malley, Phys, Rev. 162, 98 (1967).

${ }^{29}$ If the system is symmetric, $X$ will not go to 1 as $R \rightarrow \infty$, but to a matrix containing $\pm 2^{-1 / 2}$ between corresponding pairs of $g$ and $u$ states.

${ }^{30}$ S. D. Augustin, W. H. Miller, P. K. Pearson, and H. F. Schaefer III, J. Chem. Phys. 58, 2845 (1973).

${ }^{31}$ Here our terminology differs from that of Crothers (D. S. F. Crothers, Adv. Phys. 20, 405 (1971), especially p. 446]; he uses the name adiabatic representation to mean what we call the "Born-Oppenheimer"' representation. While the difference is only semantic, our usage does spare us the unedifying spectacle of adiabatic curves crossing.

${ }^{32}$ A. Dalgarno and R. McCarroll, Proc. R. Soc. (London) Ser. A 237, 383 (1956); 239, 413 (1957); see also H. Laue, J. Chem. Phys. 46, 3034 (1967); D. J. Kouri and C. F. Curtiss, J. Chem. Phys. 44, 2120 (1966).

${ }^{33}$ W. R. Thorson, J. Chem. Phys. 50, 1702 (1969).

${ }^{34}$ (a) N. Rosen and C. Zener, Phys. Rev. 40, 502 (1932); (b) D. Rapp and W. E. Francis, J. Chem. Phys. 37, 2631 (1962); (c) Yu. N. Demkov, Sov. Phys. JETP 18, 138 (1964); (d) D. S. F. Crothers, J. Phys, B 6, 1418 (1973).

${ }^{35} \mathrm{G}$. Herzberg, Spectra of Diatomic Molecules (Van Nostrand Reinhold, New York, 1950), p. $218 \mathrm{ff}$.

${ }^{36}$ F. H. Mies, Phys. Rev. A 7, 942 (1973).

${ }^{37}$ N. H. Tolk, J. C. Tully, C. W. White, J. Krauss, A. A. Monge, D. L. Simms, M. F. Robbins, S. H. Neff, and W. Lichten, Phys. Rev. A 13, 969 (1976).

${ }^{38} \mathrm{As}$ we mentioned earlier, such a representation follows automatically if the same switching function is used for all states, but it does not follow automatically if one-center ETF's are used; we must in that case assume that orthogonal states with negligible $(P+A)$ coupling can be obtained.

${ }^{39} \mathrm{It}$ might be convenient to have a name for states constructed by making $P^{R}$ vanish in a small manifold, neglecting $A^{R}$ enti rely; let's call them " $P$-diabatic." 